\title{
Projectors on the Generalized Eigenspaces for Partial Differential Equations with Time Delay
}

\author{
Arnaut Ducrot \\ Institut de Mathématiques de Bordeaux UMR CNRS 5251 \& INRIA sud-ouest Anubis, \\ Université Victor Segalen Bordeaux 2, \\ 3ter place de la victoire, Bat E 2 eme étage, 33076 Bordeaux, France \\ arnaud.ducrot@u-bordeaux2.fr \\ Pierre Magal \\ Institut de Mathématiques de Bordeaux UMR CNRS 5251 \& INRIA sud-ouest Anubis, \\ Université Victor Segalen Bordeaux 2, \\ 3ter place de la victoire, Bat E 2 eme étage, 33076 Bordeaux, France \\ pierre.magal@u-bordeaux2.fr \\ Shigui Ruan \\ Department of Mathematics, University of Miami, \\ Coral Gables, FL 33124-4250, USA \\ ruan@math.miami.edu
}

\begin{abstract}
To study the nonlinear dynamics, such as Hopf bifurcation, of partial differential equations with delay, one needs to consider the characteristic equation associated to the linearized equation and to determine the distribution of the eigenvalues; that is, to study the spectrum of the linear operator. In this paper we study the projectors on the generalized eigenspaces associated to some eigenvalues for linear partial differential equations with delay. We first rewrite partial differential equations with delay as non-densely defined semilinear Cauchy problems, then obtain formulas for the integrated solutions of the semilinear Cauchy problems with non-dense domain by using integrated semigroup theory, from which we finally derive explicit formulas for the projectors on the generalized eigenspaces associated to some eigenvalues. As examples, we apply the obtained results to study a reaction-diffusion equation with delay and an age-structured model with delay.
\end{abstract}

\section{Introduction}

Taking the interactions of spatial diffusion and time delay into account, a single species population model can be described by a partial differential equation with

1991 Mathematics Subject Classification. Primary 35K57, 34K15; Secondary 92D30.

Research of S. Ruan was partially supported by NSF grant DMS-1022728. 
time delay as follows:

$$
\left\{\begin{array}{l}
\frac{\partial u(t, x)}{\partial t}=d \frac{\partial^{2} u(t, x)}{\partial x^{2}}-a u(t-r, x)[1+u(t, x)], t>0, x \in[0, \pi], \\
\frac{\partial u(t, x)}{\partial x}=0, \quad x=0, \pi, \\
u(0, .)=u_{0} \in C([0, \pi], \mathbb{R}),
\end{array}\right.
$$

where $u(t, x)$ denotes the density of the species at time $t$ and location $x, d>0$ is the diffusion rate of the species, $r>0$ is the time delay constant, and $a>0$ is a constant. Equation (1.1) has been studied by many researchers, for example, Yoshida [55], Memory [36], and Busenberg and Huang [12] investigated Hopf bifurcation of the equation.

We consider the Banach space $Y=C([0, \pi], \mathbb{R})$ endowed with the usual supremum norm. Define $B: D(B) \subset Y \rightarrow Y$ by

$$
B \varphi=\varphi^{\prime \prime}
$$

with

Denote

$$
D(B)=\left\{\varphi \in C^{2}([0, \pi], \mathbb{R}): \varphi^{\prime}(0)=\varphi^{\prime}(\pi)=0\right\} .
$$

$$
\hat{L}(y)=-a y(-r), \quad f(y)=-a y(0) y(-r) .
$$

Equation (1.1) can be written as an abstract partial functional differential equations (PFDE) (see, for example, Travis and Webb [48, 49], Wu [54] and Faria [18]):

$$
\left\{\begin{array}{l}
\frac{d y(t)}{d t}=B y(t)+\widehat{L}\left(y_{t}\right)+f\left(t, y_{t}\right), \forall t \geq 0, \\
y_{0}=\varphi \in C_{B}
\end{array}\right.
$$

where

$$
C_{B}:=\{\varphi \in C([-r, 0] ; Y): \varphi(0) \in \overline{D(B)}\},
$$

$y_{t} \in C_{B}$ satisfies $y_{t}(\theta)=y(t+\theta), \theta \in[-r, 0], \widehat{L}: C_{B} \rightarrow Y$ is a bounded linear operator, and $f: \mathbb{R} \times C_{B} \rightarrow Y$ is a continuous map. In fact, many other partial differential equations with time delay can also be written in the form of system (1.2) (see $\mathrm{Wu}[54]$ ).

In the last 30 years, partial functional differential equations have been studied extensively by many researchers. For example, Travis and Webb [48, 49], Fitzgibbon [20], Martin and Smith [30,31], Arino and Sanchez [8] investigated the fundamental theory; Parrot [38] considered the linearized stability; Memory [37] studied the stable and unstable manifolds; Lin et al. [27], Faria et al. [19] and Adimy et al. [4] established the existence and smoothness of center manifolds; Faria [18] developed the normal form theory; Ruan et al. [41] and Ruan and Zhang [42] discussed the homoclinic bifurcation. For more detailed theories and results, we refer to the monograph of $\mathrm{Wu}[54]$.

In order to study the dynamics of system (1.2), such as Hopf bifurcation, we need to consider the characteristic equation associated to the linearized equation and to determine the distribution of the eigenvalues; i.e., to carry out the spectrum analysis of the linear operator. The aim of this article is to obtain explicit formulas for the projectors on the generalized eigenspaces associated to some eigenvalues for the linear partial functional differential equation (PFDE)

$$
\left\{\begin{array}{l}
\frac{d y(t)}{d t}=B y(t)+\widehat{L}\left(y_{t}\right), \forall t \geq 0 \\
y_{0}=\varphi \in C_{B}
\end{array}\right.
$$


In the context of ordinary functional differential equations with $Y=\mathbb{R}^{n}$ (and $B$ is bounded), this problem has been studied since the 1970s (see Hale and Verduyn Lunel [22]), the usual approach is based on the formal adjoint system. The method was recently further studied in the monograph of Diekmann et al. [14] using the so called sun star adjoint spaces, see also Kaashoek and Verduyn Lunel [24], Frasson and Verduyn Lunel [21], Diekmann et al. [13] and the references cited therein. We refer to Liu et al. [28] for a more recent study on this topic. Let us also mention that the explicit formula for the projectors on the generalized eigenspaces turns to be a crucial tool to study the bifurcations by using normal form arguments (see Liu et al [29] in the context of abstract non-densely defined Cauchy problems).

There are a few approaches to treat problem (1.2). Webb [51] and Travis and Webb $[48,49]$ viewed the problem as a nonlinear Cauchy problem and focused on many aspects using this approach. Arino and Sanchez [9] and Kappel [25] used the variation of constant formula and worked directly with the system. See also Ruess [43,44], Rhandi [40] and the references cited therein. We would like to point out that the results and techniques in the above mentioned papers do not apply directly to our problem, as we are not discussing the existence and local stability of solutions for linear partial differential equations with delay. Instead, we study the projectors on the generalized eigenspaces associated to some eigenvalues for linear partial differential equations with delay so that we can study bifurcations in such equations. Recently, Adimy [1, 2], Adimy and Arino [3], and Thieme [45] employed the integrated semigroup theory (see Ezzinbi and Adimy [17] for a survey). Here we use a formulation that is between the formulations of Adimy [1, 2] and Thieme [45] and more closely related to the one of Travis and Webb [48, 49]. See also Adimy et al. [4].

The rest of the paper is organized as follows. In section 2 we will show how to formulate the partial functional differential equation as a semilinear Cauchy problem with non-dense domain. In section 3 we recall some results on integrated semigroup theory and spectrum analysis. Section 4 presents main results on projectors on the eiganspaces. Section 5 deals with a special case for a simple eiganvalue. Some examples and discussions are given in section 6 .

\section{Preliminaries}

Let $B: D(B) \subset Y \rightarrow Y$ be a linear operator on a Banach space $\left(Y,\|\|_{Y}\right)$. Assume that it is a Hille-Yosida operator; that is, there exist two constants, $\omega_{B} \in \mathbb{R}$ and $M_{B}>0$, such that $\left(\omega_{B},+\infty\right) \subset \rho(B)$ and

$$
\left\|(\lambda I-B)^{-n}\right\| \leq \frac{M_{B}}{\left(\lambda-\omega_{B}\right)^{n}}, \forall \lambda>\omega_{B}, \forall n \geq 1 .
$$

Set

$$
Y_{0}:=\overline{D(B)} .
$$

Consider $B_{0}$, the part of $B$ in $Y_{0}$, which is defined by

$$
B_{0} y=B y \text { for each } y \in D\left(B_{0}\right)
$$

with

$$
D\left(B_{0}\right):=\left\{y \in D(B): B y \in Y_{0}\right\}
$$

For $r \geq 0$, set

$$
C:=C([-r, 0] ; Y)
$$


which is endowed with the supremum norm

$$
\|\varphi\|_{\infty}=\sup _{\theta \in[-r, 0]}\|\varphi(\theta)\|_{Y}
$$

Consider the partial functional differential equations (PFDE):

$$
\left\{\begin{array}{l}
\frac{d y(t)}{d t}=B y(t)+\widehat{L}\left(y_{t}\right)+f\left(t, y_{t}\right), \forall t \geq 0, \\
y_{0}=\varphi \in C_{B},
\end{array}\right.
$$

where $y_{t} \in C_{B}$ satisfies $y_{t}(\theta)=y(t+\theta), \theta \in[-r, 0], \widehat{L}: C_{B} \rightarrow Y$ is a bounded linear operator, and $f: \mathbb{R} \times C_{B} \rightarrow Y$ is a continuous map. Since $B$ is a Hille-Yosida operator, it is well known that $B_{0}$, the part of $B$ in $Y_{0}$, generates a $C_{0}$-semigroup of bounded linear operators $\left\{T_{B_{0}}(t)\right\}_{t>0}$ on $Y_{0}$, and $B$ generates an integrated semigroup $\left\{S_{B}(t)\right\}_{t \geq 0}$ on $Y$. The solution of the Cauchy problem (2.1) must be understood as a fixed point of

$$
y(t)=T_{B_{0}}(t) \varphi(0)+\frac{d}{d t} \int_{0}^{t} S_{B}(t-s)\left[\widehat{L}\left(y_{s}\right)+f\left(s, y_{s}\right)\right] d s .
$$

Since $\left\{T_{B_{0}}(t)\right\}_{t \geq 0}$ acts on $Y_{0}$, we observe that it is necessary to assume that

$$
\varphi(0) \in Y_{0} \Rightarrow \varphi \in C_{B} .
$$

In order to study the PFDE (2.1) by using the integrated semigroup theory, we consider the PFDE (2.1) as an abstract non-densely defined Cauchy problem. Firstly, we regard the PFDE $(2.1)$ as a PDE. Define $u \in C([0,+\infty) \times[-r, 0], Y)$ by

$$
u(t, \theta)=y(t+\theta), \forall t \geq 0, \forall \theta \in[-r, 0] .
$$

Note that if $y \in C^{1}([-r,+\infty), Y)$, then

$$
\frac{\partial u(t, \theta)}{\partial t}=y^{\prime}(t+\theta)=\frac{\partial u(t, \theta)}{\partial \theta} .
$$

Hence, we must have

$$
\frac{\partial u(t, \theta)}{\partial t}-\frac{\partial u(t, \theta)}{\partial \theta}=0, \forall t \geq 0, \forall \theta \in[-r, 0] .
$$

Moreover, for $\theta=0$, we obtain

$\frac{\partial u(t, 0)}{\partial \theta}=y^{\prime}(t)=B y(t)+\widehat{L}\left(y_{t}\right)+f\left(t, y_{t}\right)=B u(t, 0)+\widehat{L}(u(t,))+.f(t, u(t,)),. \forall t \geq 0$.

Therefore, we deduce formally that $u$ must satisfy a PDE

$$
\left\{\begin{array}{l}
\frac{\partial u(t, \theta)}{\partial t}-\frac{\partial u(t, \theta)}{\partial \theta}=0, \\
\frac{\partial u(t, 0)}{\partial \theta}=B u(t, 0)+\widehat{L}(u(t, .))+f(t, u(t, .)), \forall t \geq 0, \\
u(0, .)=\varphi \in C_{B} .
\end{array}\right.
$$

In order to rewrite the PDE (2.2) as an abstract non-densely defined Cauchy problem, we extend the state space to take into account the boundary conditions. This can be accomplished by adopting the following state space

$$
X=Y \times C
$$

taken with the usual product norm

$$
\left\|\left(\begin{array}{c}
y \\
\varphi
\end{array}\right)\right\|=\|y\|_{Y}+\|\varphi\|_{\infty} .
$$


Define the linear operator $A: D(A) \subset X \rightarrow X$ by

$$
A\left(\begin{array}{c}
0_{Y} \\
\varphi
\end{array}\right)=\left(\begin{array}{c}
-\varphi^{\prime}(0)+B \varphi(0) \\
\varphi^{\prime}
\end{array}\right), \forall\left(\begin{array}{c}
0_{Y} \\
\varphi
\end{array}\right) \in D(A)
$$

with

$$
D(A)=\left\{0_{Y}\right\} \times\left\{\varphi \in C^{1}([-r, 0], Y), \varphi(0) \in D(B)\right\} .
$$

Note that $A$ is non-densely defined because

$$
X_{0}:=\overline{D(A)}=\left\{0_{Y}\right\} \times C_{B} \neq X .
$$

We also define $L: X_{0} \rightarrow X$ by

$$
L\left(\begin{array}{c}
0_{Y} \\
\varphi
\end{array}\right):=\left(\begin{array}{c}
\widehat{L}(\varphi) \\
0_{C}
\end{array}\right)
$$

and $F: \mathbb{R} \times X_{0} \rightarrow X$ by

$$
F\left(t,\left(\begin{array}{c}
0_{Y} \\
\varphi
\end{array}\right)\right):=\left(\begin{array}{c}
f(t, \varphi) \\
0_{C}
\end{array}\right)
$$

Set

$$
v(t):=\left(\begin{array}{c}
0_{Y} \\
u(t)
\end{array}\right) .
$$

Now we can consider the PDE (2.2) as the following non-densely defined Cauchy problem

$$
\frac{d v(t)}{d t}=A v(t)+L(v(t))+F(t, v(t)), t \geq 0 ; \quad v(0)=\left(\begin{array}{c}
0_{Y} \\
\varphi
\end{array}\right) \in X_{0} .
$$

\section{Some results on integrated solutions and spectra}

In this section we will first study the integrated solutions of the Cauchy problem (2.4) in the special case

$$
\frac{d v(t)}{d t}=A v(t)+\left(\begin{array}{c}
h(t) \\
0
\end{array}\right), t \geq 0, v(0)=\left(\begin{array}{c}
0_{Y} \\
\varphi
\end{array}\right) \in X_{0}
$$

where $h \in L^{1}((0, \tau), Y)$. Recall that $v \in C([0, \tau], X)$ is an integrated solution of (3.1) if and only if

$$
\int_{0}^{t} v(s) d s \in D(A), \forall t \in[0, \tau]
$$

and

$$
v(t)=\left(\begin{array}{c}
0_{Y} \\
\varphi
\end{array}\right)+A \int_{0}^{t} v(s) d s+\int_{0}^{t}\left(\begin{array}{c}
h(s) \\
0
\end{array}\right) d s .
$$

In the sequel, we will use the integrated semigroup theory to define such an integrated solution. We refer to Arendt [5], Thieme [46], Kellermann and Hieber [26], and the book of Arendt et al. [6] for further details on this subject. We also refer to Magal and Ruan [33, 34, 35] for more results and update references.

From (3.2) we note that if $v$ is an integrated solution we must have

$$
v(t)=\lim _{h \rightarrow 0^{+}} \frac{1}{h} \int_{t}^{t+h} v(s) d s \in \overline{D(A)} .
$$

Hence

$$
v(t)=\left(\begin{array}{c}
0_{Y} \\
u(t)
\end{array}\right)
$$


with

$$
u \in C\left([0, \tau], C_{B}\right) .
$$

We introduce some notations. Let $L: D(L) \subset X \rightarrow X$ be a linear operator on a complex Banach space $X$. Denote by $\rho(L)$ the resolvent set of $L, N(L)$ the null space of $L$, and $R(L)$ the range of $L$, respectively. The spectrum of $L$ is $\sigma(L)=\mathbb{C} \backslash \rho(L)$. The point spectrum of $L$ is the set

$$
\sigma_{P}(L):=\{\lambda \in \mathbb{C}: N(\lambda I-L) \neq\{0\}\} .
$$

The essential spectrum (in the sense of Browder [11]) of $L$ is denoted by $\sigma_{\text {ess }}(L)$. That is, the set of $\lambda \in \sigma(L)$ such that at least one of the following holds: (i) $R(\lambda I-$ $L$ ) is not closed; (ii) $\lambda$ is a limit point of $\sigma(L)$; (iii) $N_{\lambda}(L):=\bigcup_{k=1}^{\infty} N\left((\lambda I-L)^{k}\right)$ is infinite dimensional. Define

$$
X_{\lambda_{0}}=\bigcup_{n \geq 0} N\left(\left(\lambda_{0}-L\right)^{n}\right) .
$$

Let $Y$ be a subspace of $X$. Then we denote by $L_{Y}: D\left(L_{Y}\right) \subset Y \rightarrow Y$ the part of $L$ on $Y$, which is defined by

$$
L_{Y} y=L y, \forall y \in D\left(L_{Y}\right):=\{y \in D(L) \cap Y: L y \in Y\} .
$$

Definition 3.1 Let $L: D(L) \subset X \rightarrow X$ be the infinitesimal generator of a linear $C_{0}$-semigroup $\left\{T_{L}(t)\right\}_{t \geq 0}$ on a Banach space $X$. We define the growth bound $\omega_{0}(L) \in[-\infty,+\infty)$ of $L$ by

$$
\omega_{0}(L):=\lim _{t \rightarrow+\infty} \frac{\ln \left(\left\|T_{L}(t)\right\|_{\mathcal{L}(X)}\right)}{t} .
$$

The essential growth bound $\omega_{0, \text { ess }}(L) \in[-\infty,+\infty)$ of $L$ is defined by

$$
\omega_{0, \mathrm{ess}}(L):=\lim _{t \rightarrow+\infty} \frac{\ln \left(\left\|T_{L}(t)\right\|_{\mathrm{ess}}\right)}{t},
$$

where $\left\|T_{L}(t)\right\|_{\text {ess }}$ is the essential norm of $T_{L}(t)$ defined by

$$
\left\|T_{L}(t)\right\|_{\mathrm{ess}}=\kappa\left(T_{L}(t) B_{X}(0,1)\right),
$$

here $B_{X}(0,1)=\left\{x \in X:\|x\|_{X} \leq 1\right\}$, and for each bounded set $B \subset X$,

$\kappa(B)=\inf \{\varepsilon>0: B$ can be covered by a finite number of balls of radius $\leq \varepsilon\}$

is the Kuratovsky measure of non-compactness.

We have the following result. The existence of the projector was first proved by Webb $[52,53]$ and the fact that there is a finite number of points of the spectrum is proved by Engel and Nagel [16, Corollary 2.1, p. 258].

Theorem 3.2 Let $L: D(L) \subset X \rightarrow X$ be the infinitesimal generator of a linear $C_{0}$-semigroup $\left\{T_{L}(t)\right\}$ on a Banach space $X$. Then

$$
\omega_{0}(L)=\max \left(\omega_{0, \mathrm{ess}}(L), \max _{\lambda \in \sigma(L) \backslash \sigma_{\text {ess }}(L)} \operatorname{Re}(\lambda)\right) .
$$

Assume in addition that $\omega_{0, \text { ess }}(L)<\omega_{0}(L)$. Then for each $\gamma \in\left(\omega_{0, \text { ess }}(L), \omega_{0}(L)\right]$, $\{\lambda \in \sigma(L): \operatorname{Re}(\lambda) \geq \gamma\} \subset \sigma_{p}(L)$ is nonempty, finite and contains only poles of the resolvent of $L$. Moreover, there exists a finite rank bounded linear operator of projection $\Pi: X \rightarrow X$ satisfying the following properties:

(a) $\Pi(\lambda-L)^{-1}=(\lambda-L)^{-1} \Pi, \forall \lambda \in \rho(L)$; 
(b) $\sigma\left(L_{\Pi(X)}\right)=\{\lambda \in \sigma(L): \operatorname{Re}(\lambda) \geq \gamma\}$;

(c) $\sigma\left(L_{(I-\Pi)(X)}\right)=\sigma(L) \backslash \sigma\left(L_{\Pi(X)}\right)$.

In Theorem 3.2 the projector $\Pi$ is the projection on the direct sum of the generalized eigenspaces of $L$ associated to all points $\lambda \in \sigma(L)$ with $\operatorname{Re}(\lambda) \geq \gamma$. As a consequence of Theorem 3.2 we have following corollary.

Corollary 3.3 Let $L: D(L) \subset X \rightarrow X$ be the infinitesimal generator of a linear $C_{0}$-semigroup $\left\{T_{L}(t)\right\}$ on a Banach space $X$, and assume that $\omega_{0, \mathrm{ess}}(L)<$ $\omega_{0}(L)$. Then

$$
\left\{\lambda \in \sigma(L): \operatorname{Re}(\lambda)>\omega_{0, \mathrm{ess}}(L)\right\} \subset \sigma_{P}(L)
$$

and each $\hat{\lambda} \in\left\{\lambda \in \sigma(L): \operatorname{Re}(\lambda)>\omega_{0, \text { ess }}(L)\right\}$ is a pole of the resolvent of $L$. That is, $\widehat{\lambda}$ is isolated in $\sigma(L)$, and there exists an integer $k_{0} \geq 1$ (the order of the pole) such that the Laurent's expansion of the resolvent takes the following form

$$
(\lambda I-L)^{-1}=\sum_{n=-k_{0}}^{\infty}\left(\lambda-\lambda_{0}\right)^{n} B_{n}^{\lambda_{0}},
$$

where $\left\{B_{n}^{\lambda_{0}}\right\}$ are bounded linear operators on $X$, and the above series converges in the norm of operators whenever $\left|\lambda-\lambda_{0}\right|$ is small enough.

The following result is due to Magal and Ruan [35, see Lemma 2.1 and Proposition 3.6].

Theorem 3.4 Let $(X,\|\|$.$) be a Banach space and L: D(L) \subset X \rightarrow X$ be a linear operator. Assume that $\rho(L) \neq \emptyset$ and $L_{0}$, the part of $L$ in $\overline{D(L)}$, is the infinitesimal generator of a linear $C_{0}$-semigroup $\left\{T_{L_{0}}(t)\right\}_{t \geq 0}$ on the Banach space $\overline{D(L)}$. Then

$$
\sigma(L)=\sigma\left(L_{0}\right)
$$

Let $X_{0}:=\overline{D(L)}, \quad \Pi_{0}: \overline{D(L)} \rightarrow \overline{D(L)}$ be a bounded linear operator of projection. Assume that

$$
\Pi_{0}\left(\lambda I-L_{0}\right)^{-1}=\left(\lambda I-L_{0}\right)^{-1} \Pi_{0}, \quad \forall \lambda>\omega
$$

and

$$
\Pi_{0}(\overline{D(L)}) \subset D\left(L_{0}\right) \text { and }\left.L_{0}\right|_{\Pi_{0}(\overline{D(L)})} \text { is bounded. }
$$

Then there exists a unique bounded linear operator of projection $\Pi$ on $X$ satisfying the following properties:

(i) $\Pi \mid \overline{D(L)}=\Pi_{0}$.

(ii) $\Pi(X) \subset \overline{D(L)}$.

(iii) $\Pi(\lambda I-L)^{-1}=(\lambda I-L)^{-1} \Pi, \forall \lambda>\omega$.

Moreover, for each $x \in X$ we have the following approximation formula

$$
\Pi x=\lim _{\lambda \rightarrow+\infty} \Pi_{0} \lambda(\lambda I-L)^{-1} x .
$$

We return to the Cauchy problem (3.1) and investigate some properties of the linear operator $A$.

Theorem 3.5 For the operator $A$ defined in (2.3), the resolvent set of $A$ satisfies

$$
\rho(A)=\rho(B) .
$$


Moreover, for each $\lambda \in \rho(A)$, we have the following explicit formula for the resolvent of $A$ :

$$
\begin{aligned}
& (\lambda I-A)^{-1}\left(\begin{array}{c}
\alpha \\
\varphi
\end{array}\right)=\left(\begin{array}{c}
0_{Y} \\
\psi
\end{array}\right) \\
& \Leftrightarrow \psi(\theta)=e^{\lambda \theta}(\lambda I-B)^{-1}[\varphi(0)+\alpha]+\int_{\theta}^{0} e^{\lambda(\theta-s)} \varphi(s) d s .
\end{aligned}
$$

Proof Let us first prove that $\rho(B) \subset \rho(A)$. If $\lambda \in \rho(B)$, for $\left(\begin{array}{c}\alpha \\ \varphi\end{array}\right) \in X$ we must find $\left(\begin{array}{c}0_{\mathbb{R}^{n}} \\ \psi\end{array}\right) \in D(A)$ such that

$$
\begin{aligned}
& (\lambda I-A)\left(\begin{array}{c}
0_{Y} \\
\psi
\end{array}\right)=\left(\begin{array}{c}
\alpha \\
\varphi
\end{array}\right) \\
& \Leftrightarrow\left\{\begin{array}{l}
\psi^{\prime}(0)-B \psi(0)=\alpha \\
\lambda \psi-\psi^{\prime}=\varphi \\
(\lambda I-B) \psi(0)=\alpha+\varphi(0) \\
\lambda \psi-\psi^{\prime}=\varphi \\
(\lambda I-B) \psi(0)=\alpha+\varphi(0)
\end{array}\right. \\
& \quad \Leftrightarrow\left\{\begin{array}{l}
\psi(\theta)=e^{\lambda(\theta-\widehat{\theta})} \psi(\widehat{\theta})+\int_{\widehat{\theta}}^{\theta} e^{\lambda(\theta-l)} \varphi(l) d l, \forall \theta \geq \widehat{\theta} \\
(\lambda I-B) \psi(0)=\alpha+\varphi(0)
\end{array}\right. \\
& \Leftrightarrow\left\{\begin{array}{l}
\psi(\widehat{\theta})=e^{\lambda \widehat{\theta}} \psi(0)-\int_{0}^{\widehat{\theta}} e^{\lambda(\widehat{\theta}-l)} \varphi(l) d l, \forall \widehat{\theta} \in[-r, 0], \\
\Leftrightarrow \psi(\widehat{\theta})=e^{\lambda \widehat{\theta}}(\lambda I-B)^{-1}[\alpha+\varphi(0)]-\int_{0}^{\hat{\theta}} e^{\lambda(\widehat{\theta}-l)} \varphi(l) d l, \forall \widehat{\theta} \in[-r, 0]
\end{array}\right.
\end{aligned}
$$

Therefore, we obtain that $\lambda \in \rho(A)$ and the formula in (3.4) holds.

It remains to prove that $\rho(A) \subset \rho(B)$, that is $\sigma(B) \subset \sigma(A)$. First, from the above computations we have the following result

$$
R(\lambda I-A)=\left\{\left(\begin{array}{c}
\alpha \\
\varphi
\end{array}\right) \in X: \quad \alpha+\varphi(0) \in R(\lambda I-B)\right\} .
$$

Moreover, we have

$$
\left(\begin{array}{c}
0_{Y} \\
\psi
\end{array}\right) \in N(\lambda I-A) \Leftrightarrow \exists y \in D(B)\left\{\begin{array}{l}
y \in N(\lambda I-B) \\
\psi(\theta)=e^{\lambda \theta} y .
\end{array}\right.
$$

Thus if $\lambda \in \sigma_{P}(B)$ (the point spectrum of $B$ ) then there exists $y \in N(\lambda I-B) \backslash\left\{0_{Y}\right\}$, and a vector $\left(\begin{array}{c}0_{Y} \\ \psi\end{array}\right) \in N(\lambda I-A) \backslash\left\{0_{C}\right\}$ with $\psi(\theta)=e^{\lambda \theta} y$. Thus $\lambda \in \sigma_{P}(A)$.

Assume that $\lambda \in \sigma(B) \backslash \sigma_{P}(B)$. Then $N(\lambda I-B)=\left\{0_{Y}\right\}$, and since $\rho(B) \neq \emptyset$, we deduce that $B$ is closed. So if $R(\lambda I-B)=Y$, by using Theorem II.20 p.30 in [10], we deduce that $(\lambda I-B)$ is invertible, that is, $(\lambda I-B)$ is a bijection from $D(B)$ into $Y$, and there exists $C>0$ such that

$$
\left\|(\lambda I-B)^{-1}\right\| \leq C
$$

so $\lambda \in \rho(B)$, a contradiction.

We deduce that $\lambda \in \sigma(B) \backslash \sigma_{P}(B)$, then $N(\lambda I-B)=\left\{0_{Y}\right\}$ and $R(\lambda I-B) \neq Y$. Thus $N(\lambda I-A)=\left\{0_{C}\right\}$. Therefore $(\lambda I-A)$ is one-to-one but not onto because of (3.1). Thus $\sigma(B) \subset \sigma(A)$ and this completes the proof of Theorem 3.5. 
Lemma 3.6 The linear operator $A: D(A) \subset X \rightarrow X$ is a Hille-Yosida operator. More precisely, for each $\omega_{A}>\omega_{B}$, there exists $M_{A} \geq 1$ such that

$$
\left\|(\lambda I-A)^{-n}\right\|_{\mathcal{L}(X)} \leq \frac{M_{A}}{\left(\lambda-\omega_{A}\right)^{n}}, \forall n \geq 1, \forall \lambda>\omega_{A} .
$$

Proof Let $\omega_{A}>\omega_{B}$. Since $B$ is a Hille-Yosida operator on $Y$, following Lemma 5.1 in Pazy [39], we can find an equivalent norm $|\cdot|_{Y}$ on $Y$ such that

$$
\left|(\lambda I-B)^{-1} x\right| \leq \frac{|x|}{\lambda-\omega_{B}} \forall \lambda>\omega_{B}, \quad \forall x \in Y .
$$

Then we define |.| the equivalent norm on $X$ by

$$
\left|\left(\begin{array}{c}
\alpha \\
\varphi
\end{array}\right)\right|=|\alpha|+\|\varphi\|_{\omega_{A}}
$$

where

$$
\|\varphi\|_{\omega_{A}}:=\sup _{\theta \in[-r, 0]}\left|e^{-\omega_{A} \theta} \varphi(\theta)\right| .
$$

Using (3.4) and the above results, we obtain for each $\lambda>\omega_{A}$ that

$$
\begin{aligned}
& \left|(\lambda I-A)^{-1}\left(\begin{array}{c}
\alpha \\
\varphi
\end{array}\right)\right| \\
\leq & \sup _{\theta \in[-r, 0]}\left[e^{-\omega_{A} \theta} e^{\lambda \theta}\left|(\lambda I-B)^{-1}[\varphi(0)+\alpha]\right|+e^{-\omega_{A} \theta} \int_{\theta}^{0} e^{\lambda(\theta-s)}|\varphi(s)| d s\right] \\
\leq & \sup _{\theta \in[-r, 0]}\left[e^{-\omega_{A} \theta} e^{\lambda \theta} \frac{1}{\lambda-\omega_{B}}[|\varphi(0)|+|\alpha|]+e^{-\omega_{A} \theta} e^{\lambda \theta} \int_{\theta}^{0} e^{-\left(\lambda-\omega_{A}\right) s} d s\|\varphi\|_{\omega_{A}}\right] \\
= & \frac{1}{\lambda-\omega_{A}}|\alpha|+\sup _{\theta \in[-r, 0]}\left[\frac{e^{-\omega_{A} \theta} e^{\lambda \theta}}{\lambda-\omega_{A}}|\varphi(0)|+\frac{e^{-\omega_{A} \theta} e^{\lambda \theta}\left[e^{-\left(\lambda-\omega_{A}\right) \theta}-1\right]}{\lambda-\omega_{A}}\|\varphi\|_{\omega_{A}}\right] \\
\leq & \frac{1}{\lambda-\omega_{A}}\left[|\alpha|+\|\varphi\|_{\omega_{A}}\right] \\
= & \frac{1}{\lambda-\omega_{A}}\left|\left(\begin{array}{c}
\alpha \\
\varphi
\end{array}\right)\right| .
\end{aligned}
$$

Therefore, (3.5) holds and the proof is completed.

Since $A$ is a Hille-Yosida operator, $A$ generates a non-degenerated integrated semigroup $\left\{S_{A}(t)\right\}_{t \geq 0}$ on $X$. It follows from Thieme [46] and Kellerman and Hieber [26] that the abstract Cauchy problem (3.1) has at most one integrated solution.

Lemma 3.7 Let $h \in L^{1}((0, \tau), Y)$ and $\varphi \in C([-r, 0], Y)$ with $\varphi(0) \in Y_{0}$. Then there exists an unique integrated solution $t \rightarrow v(t)$ of the Cauchy problem (3.1) which can be expressed explicitly by the following formula

$$
v(t)=\left(\begin{array}{c}
0_{Y} \\
u(t)
\end{array}\right)
$$

with

where

$$
u(t)(\theta)=y(t+\theta), \forall t \in[0, \tau], \forall \theta \in[-r, 0],
$$

$$
y(t)= \begin{cases}\varphi(t), & t \in[-r, 0], \\ T_{B_{0}}(t) \varphi(0)+\left(S_{B} \diamond h\right)(t), & t \in[0, \tau]\end{cases}
$$


and

$$
\left(S_{B} \diamond h\right)(t):=\frac{d}{d t}\left(S_{B} * h\right)(t),\left(S_{B} * h\right)(t):=\int_{0}^{t} S_{B}(t-s) h(s) d s .
$$

Proof Since $A$ is a Hille-Yosida operator, there is at most one integrated solution of the Cauchy problem (3.1). So it is sufficient to prove that $u$ defined by (3.6) satisfies for each $t \in[0, \tau]$ the following

$$
\left(\begin{array}{c}
0_{Y} \\
\int_{0}^{t} u(l) d l
\end{array}\right) \in D(A)
$$

and

$$
\left(\begin{array}{c}
0_{Y} \\
u(t)
\end{array}\right)=\left(\begin{array}{c}
0_{Y} \\
\varphi
\end{array}\right)+A\left(\begin{array}{c}
0_{Y} \\
\int_{0}^{t} u(l) d l
\end{array}\right)+\left(\begin{array}{c}
\int_{0}^{t} h(l) d l \\
0
\end{array}\right)
$$

Since

$$
\int_{0}^{t} u(l)(\theta) d l=\int_{0}^{t} y(l+\theta) d l=\int_{\theta}^{t+\theta} y(s) d s
$$

and $y \in C([-r, \tau], Y)$, the map $\theta \rightarrow \int_{0}^{t} u(l)(\theta) d l$ belongs to $C^{1}([-r, 0], Y)$. We also observe that

$$
\begin{aligned}
\int_{0}^{t} u(l)(0) d l & =\int_{0}^{t} y(l) d l=\int_{0}^{t} T_{B_{0}}(l) \varphi(0)+\left(S_{B} \diamond h\right)(l) d l \\
& =\int_{0}^{t} T_{B_{0}}(l) \varphi(0) d l+\left(S_{B} * h\right)(t) \in D(B)
\end{aligned}
$$

therefore, (3.7) follows. Moreover,

$$
A\left(\begin{array}{c}
0_{Y} \\
\varphi
\end{array}\right)=\left(\begin{array}{c}
-\varphi^{\prime}(0)+B \varphi(0) \\
\varphi^{\prime}
\end{array}\right)
$$

whenever $\varphi \in C^{1}([-r, 0], Y)$ with $\varphi(0) \in D(B)$. Hence

$$
\begin{aligned}
A\left(\begin{array}{c}
0 \\
\int_{0}^{t} u(l) d l
\end{array}\right) & =\left(\begin{array}{c}
-[y(t)-y(0)]+B \int_{0}^{t} y(s) d s \\
{[y(t+.)-y(.)]}
\end{array}\right) \\
& =-\left(\begin{array}{c}
0 \\
\varphi
\end{array}\right)+\left(\begin{array}{c}
-[y(t)-\varphi(0)]+B \int_{0}^{t} y(s) d s \\
y(t+.)
\end{array}\right) .
\end{aligned}
$$

Therefore, (3.8) is satisfied if and only if

$$
y(t)=\varphi(0)+B \int_{0}^{t} y(s) d s+\int_{0}^{t} h(s) d s .
$$

Since $B$ is a Hille-Yosida operator, we deduce that (3.9) is equivalent to

$$
y(t)=T_{B_{0}}(t) \varphi(0)+\left(S_{B} \diamond h\right)(t) .
$$

The proof is completed.

Recall that $A_{0}: D\left(A_{0}\right) \subset \overline{D(A)} \rightarrow \overline{D(A)}$, the part of $A$ in $\overline{D(A)}$, is defined by

$$
A_{0}\left(\begin{array}{c}
0_{Y} \\
\varphi
\end{array}\right)=A\left(\begin{array}{c}
0_{Y} \\
\varphi
\end{array}\right), \forall\left(\begin{array}{c}
0_{Y} \\
\varphi
\end{array}\right) \in D\left(A_{0}\right)
$$

where

$$
D\left(A_{0}\right)=\left\{\left(\begin{array}{c}
0_{Y} \\
\varphi
\end{array}\right) \in D(A): A\left(\begin{array}{c}
0_{Y} \\
\varphi
\end{array}\right) \in \overline{D(A)}\right\} .
$$


From the definition of $A$ in (2.3) and the fact that

$$
\overline{D(A)}=\left\{0_{Y}\right\} \times\left\{\varphi \in C([-r, 0], Y), \varphi(0) \in Y_{0}\right\},
$$

we know that $A_{0}$ is a linear operator defined by

$$
A_{0}\left(\begin{array}{c}
0_{Y} \\
\varphi
\end{array}\right)=\left(\begin{array}{c}
0_{Y} \\
\varphi^{\prime}
\end{array}\right), \forall\left(\begin{array}{c}
0_{Y} \\
\varphi
\end{array}\right) \in D\left(A_{0}\right)
$$

where

$D\left(A_{0}\right)$

$$
=\left\{\left(\begin{array}{c}
0_{Y} \\
\varphi
\end{array}\right) \in\left\{0_{Y}\right\} \times\left\{\varphi \in C^{1}([-r, 0], Y): \varphi(0) \in D(B), \quad-\varphi^{\prime}(0)+B \varphi(0)=0\right\} .\right.
$$

Now by using the fact that $A$ is a Hille-Yosida operator, we deduce that $A_{0}$ is the infinitesimal generator of a strongly continuous semigroup $\left\{T_{A_{0}}(t)\right\}_{t \geq 0}$ and

$$
v(t)=T_{A_{0}}(t)\left(\begin{array}{c}
0_{Y} \\
\varphi
\end{array}\right)
$$

is an integrated solution of

$$
\frac{d v(t)}{d t}=A v(t), t \geq 0, v(0)=\left(\begin{array}{c}
0_{Y} \\
\varphi
\end{array}\right) \in X_{0} .
$$

Using Lemma 3.7 with $h=0$, we obtain the following result.

Lemma 3.8 The linear operator $A_{0}$ is the infinitesimal generator of a strongly continuous semigroup $\left\{T_{A_{0}}(t)\right\}_{t \geq 0}$ of bounded linear operators on $\overline{D(A)}$ which is defined by

where

$$
T_{A_{0}}(t)\left(\begin{array}{c}
0_{Y} \\
\varphi
\end{array}\right)=\left(\begin{array}{c}
0_{Y} \\
\widehat{T}_{A_{0}}(t)(\varphi)
\end{array}\right),
$$

$$
\widehat{T}_{A_{0}}(t)(\varphi)(\theta)= \begin{cases}T_{B_{0}}(t+\theta) \varphi(0), & t+\theta \geq 0 \\ \varphi(t+\theta), & t+\theta \leq 0 .\end{cases}
$$

Since $A$ is a Hille-Yosida operator, we know that $A$ generates an integrated semigroup $\left\{S_{A}(t)\right\}_{t \geq 0}$ on $X$, and $t \rightarrow S_{A}(t)\left(\begin{array}{c}y \\ \varphi\end{array}\right)$ is an integrated solution of

$$
\frac{d v(t)}{d t}=A v(t)+\left(\begin{array}{c}
y \\
\varphi
\end{array}\right), t \geq 0, v(0)=0 .
$$

Since $S_{A}(t)$ is linear we have

$$
S_{A}(t)\left(\begin{array}{c}
y \\
\varphi
\end{array}\right)=S_{A}(t)\left(\begin{array}{c}
0_{Y} \\
\varphi
\end{array}\right)+S_{A}(t)\left(\begin{array}{c}
y \\
0_{C}
\end{array}\right),
$$

where

$$
S_{A}(t)\left(\begin{array}{c}
0_{Y} \\
\varphi
\end{array}\right)=\int_{0}^{t} T_{A_{0}}(l)\left(\begin{array}{c}
0_{Y} \\
\varphi
\end{array}\right) d l
$$

and $S_{A}(t)\left(\begin{array}{c}y \\ 0\end{array}\right)$ is an integrated solution of

$$
\frac{d v(t)}{d t}=A v(t)+\left(\begin{array}{l}
y \\
0
\end{array}\right), t \geq 0, v(0)=0 .
$$

Therefore, by using Lemma 3.7 with $h(t)=y$ and the above results, we obtain the following result. 
Lemma 3.9 The linear operator A generates an integrated semigroup $\left\{S_{A}(t)\right\}_{t \geq 0}$ on $X$. Moreover,

$$
S_{A}(t)\left(\begin{array}{c}
y \\
\varphi
\end{array}\right)=\left(\begin{array}{c}
0_{Y} \\
\widehat{S}_{A}(t)(y, \varphi)
\end{array}\right),\left(\begin{array}{c}
y \\
\varphi
\end{array}\right) \in X,
$$

where $\widehat{S}_{A}(t)$ is the linear operator defined by

$$
\widehat{S}_{A}(t)(y, \varphi)=\widehat{S}_{A}(t)(0, \varphi)+\widehat{S}_{A}(t)(y, 0)
$$

with

$$
\widehat{S}_{A}(t)(0, \varphi)(\theta)=\int_{0}^{t} \widehat{T}_{A_{0}}(s)(\varphi)(\theta) d s=\int_{-\theta}^{t} T_{B_{0}}(s+\theta) \varphi(0) d s+\int_{0}^{-\theta} \varphi(s+\theta) d s
$$

and

$$
\widehat{S}_{A}(t)(y, 0)(\theta)= \begin{cases}S_{B}(t+\theta) y, & t+\theta \geq 0 \\ 0, & t+\theta \leq 0 .\end{cases}
$$

Now we focus on the spectra of $A$ and $A+L$. Since $A$ is a Hille-Yosida operator, so is $A+L$. Moreover, $(A+L)_{0}: D\left((A+L)_{0}\right) \subset \overline{D(A)} \rightarrow \overline{D(A)}$, the part of $A+L$ in $\overline{D(A)}$, is a linear operator defined by

$$
(A+L)_{0}\left(\begin{array}{c}
0_{Y} \\
\varphi
\end{array}\right)=\left(\begin{array}{c}
0_{Y} \\
\varphi^{\prime}
\end{array}\right), \forall\left(\begin{array}{c}
0_{Y} \\
\varphi
\end{array}\right) \in D\left((A+L)_{0}\right)
$$

where

$$
\begin{aligned}
& D\left((A+L)_{0}\right)= \\
& \left\{\left(\begin{array}{c}
0_{Y} \\
\varphi
\end{array}\right) \in\left\{0_{Y}\right\} \times\left\{\varphi \in C^{1}([-r, 0], Y): \varphi(0) \in D(B), \varphi^{\prime}(0)=B \varphi(0)+\widehat{L}(\varphi)\right\} .\right.
\end{aligned}
$$

From Theorems 3.4 and 3.5, we know that

$$
\sigma(B)=\sigma(A)=\sigma\left(A_{0}\right) \text { and } \sigma(A+L)=\sigma\left((A+L)_{0}\right) .
$$

From (3.10), we have

$$
\widehat{T}_{A_{0}}(t)(\varphi)(\theta)=T_{B_{0}}(r+\theta) T_{B_{0}}(t-r) \varphi(0), t \geq r, \theta \in[-r, 0] .
$$

Therefore we get

$$
\omega_{0, \text { ess }}\left(A_{0}\right) \leq \omega_{0, \text { ess }}\left(B_{0}\right) .
$$

In the following lemma, we specify the essential growth rate of the $C_{0}$-semigroup generated by $(A+L)_{0}$ in some cases. Unfortunately, this problem is not fully understood.

Lemma 3.10 Assume that one of the two following properties are satisfied:

(a) For each $t>0, \widehat{L} \widehat{T}_{A_{0}}(t)$ is compact from $C$ into $Y$.

(b) For each $t>0, T_{B_{0}}(t)$ is compact on $Y$.

Then we have

$$
\omega_{0, \text { ess }}\left((A+L)_{0}\right) \leq \omega_{0, \text { ess }}\left(B_{0}\right) .
$$

Proof For Assumption (a) the result is a direct consequence of Theorem 1.2 in Ducrot et al. [15] or the results in Thieme [47]. The case (b) has been treated by Adimy et al. [4, Theorem 2.7]. 
From now on we set

$$
\Omega=\left\{\lambda \in \mathbb{C}: \operatorname{Re}(\lambda)>\omega_{0, \text { ess }}\left((A+L)_{0}\right)\right\} .
$$

From the discussion in this section, we obtain the following proposition.

Proposition 3.11 The linear operator $A+L: D(A) \subset X \rightarrow X$ is a HilleYosida operator. $(A+L)_{0}$ is the infinitesimal generator of a strongly continuous semigroup $\left\{T_{(A+L)_{0}}(t)\right\}_{t \geq 0}$ of bounded linear operators on $\overline{D(A)}$. Moreover,

$$
T_{(A+L)_{0}}(t)\left(\begin{array}{c}
0_{Y} \\
\varphi
\end{array}\right)=\left(\begin{array}{c}
0_{Y} \\
\widehat{T}_{(A+L)_{0}}(t)(\varphi)
\end{array}\right)
$$

with

$$
\widehat{T}_{(A+L)_{0}}(t)(\varphi)(\theta)=y(t+\theta), \forall t \geq 0, \forall \theta \in[-r, 0],
$$

where

$$
y(t)= \begin{cases}\varphi(t), & \forall t \in[-r, 0], \\ T_{B_{0}}(t) \varphi(0)+\left(S_{B} \diamond \widehat{L}(y .)\right)(t), & \forall t \geq 0 .\end{cases}
$$

Furthermore, we have that

$$
\sigma\left((A+L)_{0}\right) \cap \Omega=\sigma_{P}\left((A+L)_{0}\right) \cap \Omega=\{\lambda \in \Omega: \quad N(\Delta(\lambda)) \neq 0\},
$$

where $\Delta(\lambda): D(B) \subset Y \rightarrow Y$ is the following linear operator

$$
\Delta(\lambda)=\lambda I-B-\widehat{L}\left(e^{\lambda \cdot} I_{Y}\right) .
$$

Then each $\lambda_{0} \in \sigma\left((A+L)_{0}\right) \cap \Omega$ is a pole in $\Omega$ of $(\lambda I-(A+L))^{-1}$. For each $\gamma>\omega_{0, \mathrm{ess}}\left((A+L)_{0}\right)$, the subset $\left\{\lambda \in \sigma\left((A+L)_{0}\right): \operatorname{Re}(\lambda) \geq \gamma\right\}$ is either empty or finite.

Proof The first part of the result follows immediately from Lemma 3.7 applied with $h(t)=\widehat{L}\left(y_{t}\right)$, and the last part of the proof follows from Theorem 3.2, Corollary 3.3, and Theorem 3.4.

\section{Projectors on the eigenspaces}

Let $\lambda_{0} \in \sigma(A+L) \cap \Omega$. From the above discussion we already knew that $\lambda_{0}$ is a pole of $(\lambda I-(A+L))^{-1}$ of finite order $k_{0} \geq 1$. This means that $\lambda_{0}$ is isolated in $\sigma(A+L)$ and the Laurent's expansion of the resolvent around $\lambda_{0}$ takes the following form

$$
(\lambda I-(A+L))^{-1}=\sum_{n=-k_{0}}^{+\infty}\left(\lambda-\lambda_{0}\right)^{n} B_{n}^{\lambda_{0}} .
$$

The bounded linear operator $B_{-1}^{\lambda_{0}}$ is the projector on the generalized eigenspace of $(A+L)$ associated to $\lambda_{0}$. The goal of this section is to provide a method to compute $B_{-1}^{\lambda_{0}}$. We remark that

$$
\left(\lambda-\lambda_{0}\right)^{k_{0}}(\lambda I-(A+L))^{-1}=\sum_{m=0}^{+\infty}\left(\lambda-\lambda_{0}\right)^{m} B_{m-k_{0}}^{\lambda_{0}} .
$$

So we have the following approximation formula

$$
B_{-1}^{\lambda_{0}}=\lim _{\lambda \rightarrow \lambda_{0}} \frac{1}{\left(k_{0}-1\right) !} \frac{d^{k_{0}-1}}{d \lambda^{k_{0}-1}}\left(\left(\lambda-\lambda_{0}\right)^{k_{0}}(\lambda I-(A+L))^{-1}\right) .
$$


In order to compute an explicit formula for the resolvent of $A+L$ we will use the following lemma.

Lemma 4.1 Let $A: D(A) \subset X \rightarrow X$ be a linear operator on a Banach space $X$ with $\rho(A) \neq \emptyset$. Let $B: \overline{D(A)} \rightarrow X$ be a bounded linear operator. Then for each $\lambda \in \rho(A)$ we have

$$
\lambda \in \rho(A+B) \Leftrightarrow 1 \in \rho\left(B(\lambda I-A)^{-1}\right) .
$$

Moreover, for each $\lambda \in \rho(A+B)$ we have

$$
\begin{gathered}
(\lambda I-A-B)^{-1}=(\lambda I-A)^{-1}\left[I-B(\lambda I-A)^{-1}\right]^{-1} \\
{\left[I-B(\lambda I-A)^{-1}\right]^{-1}=I+B(\lambda I-A-B)^{-1}}
\end{gathered}
$$

Proof Assume first that $1 \in \rho\left(B(\lambda I-A)^{-1}\right)$. Then

$$
\begin{aligned}
& (\lambda I-A-B)(\lambda I-A)^{-1}\left[I-B(\lambda I-A)^{-1}\right]^{-1} \\
& \quad=\left[I-B(\lambda I-A)^{-1}\right]\left[I-B(\lambda I-A)^{-1}\right]^{-1}=I,
\end{aligned}
$$

and

$$
\begin{aligned}
& (\lambda I-A)^{-1}\left[I-B(\lambda I-A)^{-1}\right]^{-1}(\lambda I-A-B) \\
& \quad=(\lambda I-A)^{-1}\left[I-B(\lambda I-A)^{-1}\right]^{-1}\left(I-B(\lambda I-A)^{-1}\right)(\lambda I-A) \\
& \quad=I_{D(A)} .
\end{aligned}
$$

Thus $\lambda \in \rho(A+B)$, and

$$
(\lambda I-A-B)^{-1}=(\lambda I-A)^{-1}\left[I-B(\lambda I-A)^{-1}\right]^{-1} .
$$

Conversely, assume that $\lambda \in \rho(A+B)$, then

$$
\begin{aligned}
{[I} & \left.-B(\lambda I-A)^{-1}\right]\left[I+B(\lambda I-A-B)^{-1}\right] \\
& =\left[I-B(\lambda I-A)^{-1}\right]\left[(\lambda I-A-B)(\lambda I-A-B)^{-1}+B(\lambda I-A-B)^{-1}\right] \\
& =\left[I-B(\lambda I-A)^{-1}\right]\left[(\lambda I-A)(\lambda I-A-B)^{-1}\right] \\
& =(\lambda I-A)(\lambda I-A-B)^{-1}-B(\lambda I-A-B)^{-1}=I
\end{aligned}
$$

and

$$
\begin{aligned}
{[I} & \left.+B(\lambda I-A-B)^{-1}\right]\left[I-B(\lambda I-A)^{-1}\right] \\
& =\left[(\lambda I-A)(\lambda I-A-B)^{-1}\right]\left[I-B(\lambda I-A)^{-1}\right] \\
& =(\lambda I-A)(\lambda I-A-B)^{-1}[\lambda I-A-B](\lambda I-A)^{-1} \\
& =I .
\end{aligned}
$$

Thus, $1 \in \rho\left(B(\lambda I-A)^{-1}\right)$ and

$$
\left[I-B(\lambda I-A)^{-1}\right]^{-1}=I+B(\lambda I-A-B)^{-1} .
$$


This completes the proof.

In order to give an explicit formula for $B_{-1}^{\lambda_{0}}$, we need the following results.

Lemma 4.2 We have the following equivalence:

$$
\lambda \in \rho(A+L) \cap \Omega \Leftrightarrow \Delta(\lambda) \text { is invertible. }
$$

Moreover, we have the following explicit formula for the resolvent of $A+L$

$$
\begin{aligned}
& (\lambda I-(A+L))^{-1}\left(\begin{array}{c}
\alpha \\
\varphi
\end{array}\right)=\left(\begin{array}{c}
0_{Y} \\
\psi
\end{array}\right) \\
& \Leftrightarrow \\
& \psi(\theta)=\int_{\theta}^{0} e^{\lambda(\theta-s)} \varphi(s) d s+e^{\lambda \theta} \Delta(\lambda)^{-1}\left[\alpha+\varphi(0)+\widehat{L}\left(\int_{0}^{0} e^{\lambda(.-s)} \varphi(s) d s\right)\right] .
\end{aligned}
$$

Proof We consider the linear operator $A_{\gamma}: D(A) \subset X \rightarrow X$ defined by

$$
A_{\gamma}\left(\begin{array}{c}
0_{Y} \\
\varphi
\end{array}\right)=\left(\begin{array}{c}
-\varphi^{\prime}(0)+(B-\gamma I) \varphi(0) \\
\varphi^{\prime}
\end{array}\right), \forall\left(\begin{array}{c}
0_{Y} \\
\varphi
\end{array}\right) \in D(A)
$$

and

$$
L_{\gamma}\left(\begin{array}{c}
0_{Y} \\
\varphi
\end{array}\right)=\left(\begin{array}{c}
\widehat{L}(\varphi)+\gamma \varphi(0) \\
0_{C}
\end{array}\right)
$$

Then we have

$$
A+L=A_{\gamma}+L_{\gamma}
$$

Moreover,

$$
\omega_{0}\left(B_{0}-\gamma I\right)=\omega_{0}\left(B_{0}\right)-\gamma
$$

Hence by Theorem 3.5, for $\lambda \in \mathbb{C}$ with $\operatorname{Re}(\lambda)>\omega_{0}\left(B_{0}\right)-\gamma$, we have $\lambda \in \rho\left(A_{\gamma}\right)$ and

$$
\begin{aligned}
& \left(\lambda I-A_{\gamma}\right)^{-1}\left(\begin{array}{c}
\alpha \\
\varphi
\end{array}\right)=\left(\begin{array}{c}
0_{Y} \\
\psi
\end{array}\right) \\
& \Leftrightarrow \psi(\theta)=e^{\lambda \theta}(\lambda I-(B-\gamma I))^{-1}[\varphi(0)+\alpha]+\int_{\theta}^{0} e^{\lambda(\theta-s)} \varphi(s) d s .
\end{aligned}
$$

Therefore, for each $\lambda \in \mathbb{C}$ with $\operatorname{Re}(\lambda)>\omega_{0}\left(B_{0}\right)-\gamma$, by Lemma 4.1 we deduce that $\left[\lambda I-\left(A_{\gamma}+L_{\gamma}\right)\right]$ is invertible if and only if $I-L_{\gamma}\left(\lambda I-A_{\gamma}\right)^{-1}$ is invertible, and

$$
\left(\lambda I-\left(A_{\gamma}+L_{\gamma}\right)\right)^{-1}=\left(\lambda I-A_{\gamma}\right)^{-1}\left[I-L_{\gamma}\left(\lambda I-A_{\gamma}\right)^{-1}\right]^{-1} .
$$

We also know that $\left[I-L_{\gamma}\left(\lambda I-A_{\gamma}\right)^{-1}\right]\left(\begin{array}{c}\alpha \\ \varphi\end{array}\right)=\left(\begin{array}{c}\widehat{\alpha} \\ \widehat{\varphi}\end{array}\right)$ is equivalent to $\varphi=\widehat{\varphi}$ and

$$
\begin{aligned}
& \alpha-\left[\widehat{L}\left(e^{\lambda \cdot}(\lambda I-(B-\gamma I))^{-1} \alpha\right)+\gamma(\lambda I-(B-\gamma I))^{-1} \alpha\right] \\
& =\widehat{\alpha}+\left[\begin{array}{c}
\widehat{L}\left(e^{\lambda \cdot}(\lambda I-(B-\gamma I))^{-1} \widehat{\varphi}(0)+\int_{\cdot}^{0} e^{\lambda(.-s)} \widehat{\varphi}(s) d s\right) \\
+\gamma(\lambda I-(B-\gamma I))^{-1} \widehat{\varphi}(0)
\end{array}\right] .
\end{aligned}
$$

Because

$$
\begin{aligned}
\alpha & -\widehat{L}\left(e^{\lambda \cdot}(\lambda I-(B-\gamma I))^{-1} \alpha\right)-\gamma(\lambda I-(B-\gamma I))^{-1} \alpha \\
& =\left[\lambda I-(B-\gamma I)-\widehat{L}\left(e^{\lambda \cdot I}\right)-\gamma I\right](\lambda I-(B-\gamma I))^{-1} \alpha \\
& =\left[\lambda I-B-\widehat{L}\left(e^{\lambda \cdot I}\right)\right](\lambda I-(B-\gamma I))^{-1} \alpha
\end{aligned}
$$




$$
=\Delta(\lambda)(\lambda I-(B-\gamma I))^{-1} \alpha
$$

and $B$ is closed, we deduce that $\Delta(\lambda)$ is closed, and by using the same arguments as in the proof of Theorem 3.5 to

$$
\begin{aligned}
& \Delta(\lambda)(\lambda I-(B-\gamma I))^{-1} \alpha \\
& \quad=\widehat{\alpha}+\left[\begin{array}{c}
\widehat{L}\left(e^{\lambda \cdot}(\lambda I-(B-\gamma I))^{-1} \widehat{\varphi}(0)+\int^{0}{ }^{0} e^{\lambda(.-s)} \widehat{\varphi}(s) d s\right) \\
+\gamma(\lambda I-(B-\gamma I))^{-1} \widehat{\varphi}(0)
\end{array}\right]
\end{aligned}
$$

we deduce that $\left[I-L_{\gamma}\left(\lambda I-A_{\gamma}\right)^{-1}\right]$ is invertible if and only if

$$
\Delta(\lambda)=\left[\lambda I-B-\widehat{L}\left(e^{\lambda} I\right)\right]
$$

is invertible. So for $\lambda \in \Omega,[\lambda I-(A+L)]$ is invertible if and only if $\Delta(\lambda)$ is invertible.

Moreover,

$$
\left[I-L_{\gamma}\left(\lambda I-A_{\gamma}\right)^{-1}\right]^{-1}\left(\begin{array}{c}
\widehat{\alpha} \\
\widehat{\varphi}
\end{array}\right)=\left(\begin{array}{l}
\alpha \\
\varphi
\end{array}\right)
$$

is equivalent to $\varphi=\widehat{\varphi}$ and

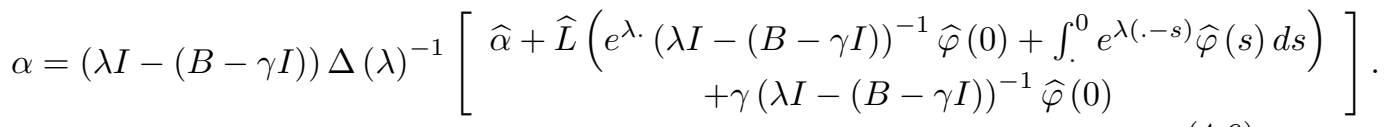

Note that $A+L=A_{\gamma}+L_{\gamma}$. By using (4.4), (4.5) and (4.6), we obtain for each $\gamma>0$ large enough that

$$
\begin{aligned}
& (\lambda I-(A+L))^{-1}\left(\begin{array}{c}
\alpha \\
\varphi
\end{array}\right)=\left(\begin{array}{c}
0_{\mathbb{R}^{n}} \\
\psi
\end{array}\right) \\
& \Leftrightarrow \\
& \psi(\theta)=e^{\lambda \theta}(\lambda I-(B-\gamma I))^{-1} \varphi(0)+\int_{\theta}^{0} e^{\lambda(\theta-s)} \varphi(s) d s \\
& +e^{\lambda \theta} \Delta(\lambda)^{-1}\left[\begin{array}{r}
\alpha+\widehat{L}\left(e^{\lambda \cdot}(\lambda I-(B-\gamma I))^{-1} \varphi(0)+\int^{0} e^{\lambda(.-s)} \varphi(s) d s\right) \\
+\gamma(\lambda I-(B-\gamma I))^{-1} \varphi(0)
\end{array}\right],
\end{aligned}
$$

but

$$
\begin{aligned}
(\lambda I- & (B-\gamma I))^{-1} \varphi(0)+\Delta(\lambda)^{-1}\left[\widehat{L}\left(e^{\lambda \cdot I}\right)+\gamma I\right](\lambda I-(B-\gamma I))^{-1} \varphi(0) \\
= & \left(I+\left[\lambda I-B-\widehat{L}\left(e^{\lambda \cdot} I\right)\right]^{-1}\left[\widehat{L}\left(e^{\lambda \cdot} I\right)+B-\lambda I+\lambda I-(B-\gamma I)\right]\right) \\
& \cdot(\lambda I-(B-\gamma I))^{-1} \varphi(0) \\
= & \left(I-I+\left[\lambda I-B-\widehat{L}\left(e^{\lambda \cdot I}\right]^{-1}[\lambda I-(B-\gamma I)]\right)(\lambda I-(B-\gamma I))^{-1} \varphi(0)\right. \\
= & {\left[\lambda I-B-\widehat{L}\left(e^{\lambda \cdot} I\right)\right]^{-1} \varphi(0) }
\end{aligned}
$$

and the result follows.

Next we introduce the following operators $\widetilde{\Pi}: X_{0} \rightarrow C$ and $F: Y \rightarrow X$ such that

Then from Lemma 4.2 we have:

$$
\widetilde{\Pi}\left(\begin{array}{c}
0_{Y} \\
\varphi
\end{array}\right)=\varphi(0), \quad F \alpha=\left(\begin{array}{c}
\alpha \\
0_{C}
\end{array}\right) .
$$

$$
\widetilde{\Pi}(\lambda I-(A+L))^{-1} F \alpha=\Delta^{-1}(\lambda) \alpha, \quad \forall \lambda \in \rho(A+L) \cap \Omega, \quad \forall \alpha \in Y .
$$


Since $\lambda \rightarrow(\lambda I-(A+L))^{-1}$ is holomorphic from $\Omega$ into $\mathcal{L}(X)$, we deduce from the above formula that the map $\lambda \rightarrow \Delta^{-1}(\lambda)$ is holomorphic in $\Omega$. Moreover, by Proposition 3.11 we know that $\Delta^{-1}$ (.) has only finite order poles. Therefore, $\Delta^{-1}(\lambda)$ has a Laurent's expansion around $\lambda_{0}$ as follows

$$
\Delta(\lambda)^{-1}=\sum_{n=-\widehat{k}_{0}}^{+\infty}\left(\lambda-\lambda_{0}\right)^{n} \Delta_{n}, \quad \Delta_{n} \in \mathcal{L}(Y), \quad \forall n \geq-\widehat{k}_{0} .
$$

From the following lemma we know that $\widehat{k}_{0}=k_{0}$.

Lemma 4.3 Let $\lambda_{0} \in \sigma(A+L) \cap \Omega$. Then the following statements are equivalent

(a) $\lambda_{0}$ is a pole of order $k_{0}$ of $(\lambda I-(A+L))^{-1}$.

(b) $\lambda_{0}$ is a pole of order $k_{0}$ of $\Delta(\lambda)^{-1}$.

(c) $\lim _{\lambda \rightarrow \lambda_{0}}\left(\lambda-\lambda_{0}\right)^{k_{0}} \Delta(\lambda)^{-1} \neq 0$ and $\lim _{\lambda \rightarrow \lambda_{0}}\left(\lambda-\lambda_{0}\right)^{k_{0}+1} \Delta(\lambda)^{-1}=0$.

Moreover, if one the above assertions is satisfied, then for each $n \geq-k_{0}$,

$$
R\left(\Delta_{n}\right) \subset D(B)
$$

and

$$
B \Delta_{n} \in \mathcal{L}(Y) .
$$

Proof The proof of the equivalence follows from the explicit formula of the resolvent of $A+L$ obtained in Lemma 4.2. It remains to prove the last part of the lemma. Let $\lambda_{0} \in \sigma(A+L) \cap \Omega$ be a pole of order $k_{0}$ of the resolvent of $\lambda \rightarrow \Delta(\lambda)^{-1}$. Let $\gamma \in \rho(B)$. Then by Proposition 3.11, $\lambda \in \rho(A+L) \cap \Omega \Leftrightarrow \Delta(\lambda)$ is invertible. But

with

$$
\Delta(\lambda)=\gamma I-B+C_{\gamma}(\lambda)
$$

$$
C_{\gamma}(\lambda)=\left(\lambda I-\gamma I-\widehat{L}\left(e^{\lambda \cdot} I\right)\right)
$$

So by Lemma 4.1, if $\Delta(\lambda)$ is invertible then $1 \in \rho\left(I-C_{\gamma}(\lambda)(\gamma I-B)^{-1}\right)$ and

$$
\Delta(\lambda)^{-1}=(\gamma I-B)^{-1}\left[I-C_{\gamma}(\lambda)(\gamma I-B)^{-1}\right]^{-1} .
$$

Clearly $\lambda \rightarrow C_{\gamma}(\lambda)$ is holomorphic, $\lambda_{0}$ is pole of order $k_{0}$. It follows that

$$
\left[I-C_{\gamma}(\lambda)(\gamma I-B)^{-1}\right]^{-1}=\sum_{n=-k_{0}}^{+\infty}\left(\lambda-\lambda_{0}\right)^{n} \widetilde{\Delta}_{n}^{\gamma}
$$

and by the uniqueness of the Laurent's expension we obtain

$$
\Delta_{n}=(\gamma I-B)^{-1} \widetilde{\Delta}_{n}^{\gamma}, \forall n \geq-k_{0} .
$$

This completes the proof.

Lemma 4.4 The operators $\Delta_{-1}, \ldots, \Delta_{-k_{0}}$ satisfy

$$
\Delta_{k_{0}}\left(\lambda_{0}\right)\left(\begin{array}{c}
\Delta_{-1} \\
\Delta_{-2} \\
\vdots \\
\Delta_{-k_{0}+1} \\
\Delta_{-k_{0}}
\end{array}\right)=\left(\begin{array}{c}
0 \\
\vdots \\
0
\end{array}\right)
$$


and

$$
\left(\begin{array}{ccccc}
\Delta_{-k_{0}} & \Delta_{-k_{0}+1} & \cdots & \Delta_{-2} & \Delta_{-1}
\end{array}\right) \Delta_{k_{0}}\left(\lambda_{0}\right)=\left(\begin{array}{ccc}
0 & \cdots & 0
\end{array}\right),
$$

where $\Delta_{k_{0}}\left(\lambda_{0}\right)$ is the following operator matrix (from $D(B)^{k_{0}}$ into $Y^{k_{0}}$ )

$\Delta_{k_{0}}\left(\lambda_{0}\right)=\left(\begin{array}{ccccc}\Delta^{(0)}\left(\lambda_{0}\right) & \Delta^{(1)}\left(\lambda_{0}\right) & \Delta^{(2)}\left(\lambda_{0}\right) / 2 ! & \cdots & \Delta^{\left(k_{0}-1\right)}\left(\lambda_{0}\right) /\left(k_{0}-1\right) ! \\ 0 & \ddots & \ddots & \ddots & \vdots \\ \vdots & 0 & \ddots & \ddots & \Delta^{(2)}\left(\lambda_{0}\right) / 2 ! \\ \vdots & & \ddots & \ddots & \Delta^{(1)}\left(\lambda_{0}\right) \\ 0 & \cdots & \cdots & 0 & \Delta^{(0)}\left(\lambda_{0}\right)\end{array}\right)$,

where

$$
\Delta^{(0)}(\lambda)=\Delta(\lambda)=\lambda I-B-\widehat{L}\left(e^{\lambda} \cdot I_{Y}\right)
$$

and

$$
\Delta^{(n)}(\lambda)=\frac{d^{n}}{d \lambda^{n}}\left(\lambda I-\widehat{L}\left(e^{\lambda \cdot} I\right)\right), \forall n \geq 1 .
$$

Proof We have

$$
\left(\lambda-\lambda_{0}\right)^{k_{0}} I=\Delta(\lambda)\left(\sum_{n=0}^{+\infty}\left(\lambda-\lambda_{0}\right)^{n} \Delta_{n-k_{0}}\right)=\left(\sum_{n=0}^{+\infty}\left(\lambda-\lambda_{0}\right)^{n} \Delta_{n-k_{0}}\right) \Delta(\lambda)
$$

and

$$
\Delta(\lambda)=\Delta\left(\lambda_{0}\right)+\left[\left(\lambda-\lambda_{0}\right) I-\left(\widehat{L}\left(e^{\lambda \cdot I} I\right)-\widehat{L}\left(e^{\lambda_{0} \cdot} I\right)\right)\right]
$$

So

$$
\Delta(\lambda)=\Delta\left(\lambda_{0}\right)+\sum_{n=1}^{+\infty}\left(\lambda-\lambda_{0}\right)^{n} \frac{\Delta^{(n)}\left(\lambda_{0}\right)}{n !}
$$

Hence,

$$
\left(\lambda-\lambda_{0}\right)^{k_{0}} I=\left(\sum_{n=0}^{+\infty}\left(\lambda-\lambda_{0}\right)^{n} \frac{\Delta^{(n)}\left(\lambda_{0}\right)}{n !}\right)\left(\sum_{n=0}^{+\infty}\left(\lambda-\lambda_{0}\right)^{n} \Delta_{n-k_{0}}\right) .
$$

By using the last part of Lemma 4.3, we know that for each $n \geq-k_{0}, \Delta^{(0)}\left(\lambda_{0}\right) \Delta_{n}=$ $\Delta\left(\lambda_{0}\right) \Delta_{n}$ is bounded and linear for $Y$ into itself, so we obtain

$$
\left(\lambda-\lambda_{0}\right)^{k_{0}} I=\sum_{n=0}^{+\infty}\left(\lambda-\lambda_{0}\right)^{n} \sum_{k=0}^{n} \frac{\Delta^{(n-k)}\left(\lambda_{0}\right)}{(n-k) !} \Delta_{k-k_{0}}
$$

and

$$
\left(\lambda-\lambda_{0}\right)^{k_{0}} I=\sum_{n=0}^{+\infty}\left(\lambda-\lambda_{0}\right)^{n} \sum_{k=0}^{n} \Delta_{k-k_{0}} \frac{\Delta^{(n-k)}\left(\lambda_{0}\right)}{(n-k) !} .
$$

By the uniqueness of the Taylor's expansion for analytic maps, we obtain that for $n \in\left\{0, \ldots, k_{0}-1\right\}$,

$$
0=\sum_{k=0}^{n} \Delta_{k-k_{0}} \frac{\Delta^{(n-k)}\left(\lambda_{0}\right)}{(n-k) !}=\sum_{k=0}^{n} \frac{\Delta^{(n-k)}\left(\lambda_{0}\right)}{(n-k) !} \Delta_{k-k_{0}} .
$$

Therefore, the result follows. 
Now we look for an explicit formula for the projector $B_{-1}^{\lambda_{0}}$ on the generalized eigenspace associated to $\lambda_{0}$. Set

$$
\Psi_{1}(\lambda)(\varphi)(\theta):=\int_{\theta}^{0} e^{\lambda(\theta-s)} \varphi(s) d s
$$

and

$$
\Psi_{2}(\lambda)\left(\left(\begin{array}{c}
\alpha \\
\varphi
\end{array}\right)\right)(\theta):=e^{\lambda \theta}\left[\alpha+\varphi(0)+\widehat{L}\left(\int^{0} e^{\lambda(\cdot-s)} \varphi(s) d s\right)\right] .
$$

Then both maps are analytic and

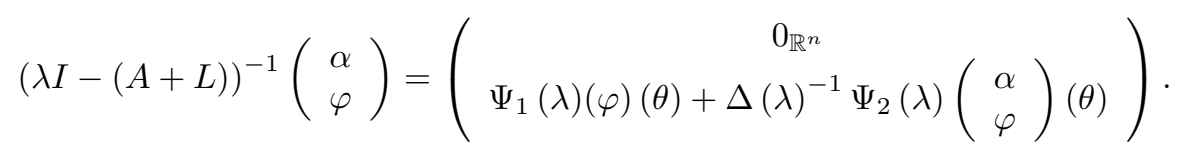

We observe that the only singularity in the last expression is $\Delta(\lambda)^{-1}$. Since $\Psi_{1}$ and $\Psi_{2}$ are analytic, we have for $j=1,2$ that

$$
\Psi_{j}(\lambda)=\sum_{n=0}^{+\infty} \frac{\left(\lambda-\lambda_{0}\right)^{n}}{n !} L_{n}^{j}\left(\lambda_{0}\right)
$$

where $\left|\lambda-\lambda_{0}\right|$ is small enough and $L_{n}^{j}():.=\frac{d^{n} \Psi_{j}(.)}{d \lambda^{n}}, \forall n \geq 0, \forall j=1,2$. Hence, we get

$$
\begin{aligned}
\lim _{\lambda \rightarrow \lambda_{0}} & \frac{1}{\left(k_{0}-1\right) !} \frac{d^{k_{0}-1}}{d \lambda^{k_{0}-1}}\left(\left(\lambda-\lambda_{0}\right)^{k_{0}} \Psi_{1}(\lambda)\right) \\
\quad & =\lim _{\lambda \rightarrow \lambda_{0}} \frac{1}{\left(k_{0}-1\right) !} \sum_{n=0}^{+\infty} \frac{\left(n+k_{0}\right) !}{(n+1) !} \frac{\left(\lambda-\lambda_{0}\right)^{n+1}}{n !} L_{n}^{1}\left(\lambda_{0}\right) \\
\quad & =0
\end{aligned}
$$

and

$$
\begin{aligned}
& \lim _{\lambda \rightarrow \lambda_{0}} \frac{1}{\left(k_{0}-1\right) !} \frac{d^{k_{0}-1}}{d \lambda^{k_{0}-1}}\left(\lambda-\lambda_{0}\right)^{k_{0}} \Delta(\lambda)^{-1} \Psi_{2}(\lambda) \\
= & \lim _{\lambda \rightarrow \lambda_{0}} \frac{1}{\left(k_{0}-1\right) !} \frac{d^{k_{0}-1}}{d \lambda^{k_{0}-1}}\left[\left(\sum_{n=-k_{0}}^{+\infty}\left(\lambda-\lambda_{0}\right)^{n+k_{0}} \Delta_{n}\right)\left(\sum_{n=0}^{+\infty} \frac{\left(\lambda-\lambda_{0}\right)^{n}}{n !} L_{n}^{2}\left(\lambda_{0}\right)\right)\right] \\
= & \lim _{\lambda \rightarrow \lambda_{0}} \frac{1}{\left(k_{0}-1\right) !} \frac{d^{k_{0}-1}}{d \lambda^{k_{0}-1}}\left[\left(\sum_{n=0}^{+\infty}\left(\lambda-\lambda_{0}\right)^{n} \Delta_{n-k_{0}}\right)\left(\sum_{n=0}^{+\infty} \frac{\left(\lambda-\lambda_{0}\right)^{n}}{n !} L_{n}^{2}\left(\lambda_{0}\right)\right)\right] \\
= & \lim _{\lambda \rightarrow \lambda_{0}} \frac{1}{\left(k_{0}-1\right) !} \frac{d^{k_{0}-1}}{d \lambda^{k_{0}-1}}\left[\sum_{n=0}^{+\infty} \sum_{j=0}^{n}\left(\lambda-\lambda_{0}\right)^{n-j} \Delta_{n-j-k_{0}} \frac{\left(\lambda-\lambda_{0}\right)^{j}}{j !} L_{j}^{2}\left(\lambda_{0}\right)\right] \\
= & \lim _{\lambda \rightarrow \lambda_{0}} \frac{1}{\left(k_{0}-1\right) !} \frac{d^{k_{0}-1}}{d \lambda^{k_{0}-1}}\left[\sum_{n=0}^{+\infty}\left(\lambda-\lambda_{0}\right)^{n} \sum_{j=0}^{n} \Delta_{n-j-k_{0}} \frac{1}{j !} L_{j}^{2}\left(\lambda_{0}\right)\right] \\
= & \sum_{j=0}^{k_{0}-1} \frac{1}{j !} \Delta_{-1-j} L_{j}^{2}\left(\lambda_{0}\right) .
\end{aligned}
$$


From the above results we can obtain the explicit formula for the projector $B_{-1}^{\lambda_{0}}$ on the generalized eigenspace associated to $\lambda_{0}$, which is given in the following proposition.

Proposition 4.5 Each $\lambda_{0} \in \sigma((A+L))$ with $\operatorname{Re}\left(\lambda_{0}\right)>\omega_{0, \mathrm{ess}}\left((A+L)_{0}\right)$ is a pole of $(\lambda I-(A+L))^{-1}$ of order $k_{0} \geq 1$. Moreover, $k_{0}$ is the only integer such that there exists $\Delta_{-k_{0}} \in \mathcal{L}(Y)$ with $\Delta_{-k_{0}} \neq 0$, such that

$$
\Delta_{-k_{0}}=\lim _{\lambda \rightarrow \lambda_{0}}\left(\lambda-\lambda_{0}\right)^{k_{0}} \Delta(\lambda)^{-1} .
$$

Furthermore, the projector $B_{-1}^{\lambda_{0}}$ on the generalized eigenspace of $(A+L)$ associated to $\lambda_{0}$ is defined by the following formula

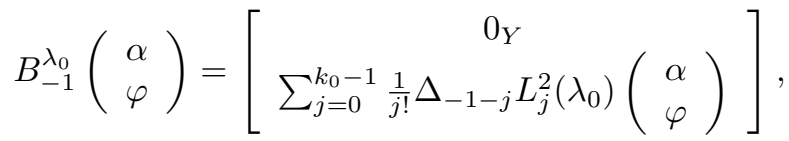

where

and

$$
\begin{gathered}
\Delta_{-j}=\lim _{\lambda \rightarrow \lambda_{0}} \frac{1}{\left(k_{0}-j\right) !} \frac{d^{k_{0}-j}}{d \lambda^{k_{0}-j}}\left(\left(\lambda-\lambda_{0}\right)^{k_{0}} \Delta(\lambda)^{-1}\right), j=1, \ldots, k_{0}, \\
L_{0}^{2}(\lambda)\left(\begin{array}{c}
\alpha \\
\varphi
\end{array}\right)=e^{\lambda \theta}\left[\alpha+\varphi(0)+\widehat{L}\left(\int^{0} e^{\lambda(.-s)} \varphi(s) d s\right)\right],
\end{gathered}
$$

$$
\begin{aligned}
L_{j}^{2}(\lambda)\left(\begin{array}{c}
\alpha \\
\varphi
\end{array}\right) & =\frac{d^{j}}{d \lambda^{j}}\left[L_{0}^{2}(\lambda)\left(\begin{array}{c}
\alpha \\
\varphi
\end{array}\right)\right] \\
& =\sum_{k=0}^{j} C_{j}^{k} \theta^{k} e^{\lambda \theta} \frac{d^{j-k}}{d \lambda^{j-k}}\left[\alpha+\varphi(0)+\widehat{L}\left(\int^{0} e^{\lambda(.-s)} \varphi(s) d s\right)\right], j \geq 1,
\end{aligned}
$$

in which

$\frac{d^{i}}{d \lambda^{i}}\left[\alpha+\varphi(0)+\widehat{L}\left(\int_{.}^{0} e^{\lambda(.-s)} \varphi(s) d s\right)\right]=\widehat{L}\left(\int_{.}^{0}(.-s)^{i} e^{\lambda(.-s)} \varphi(s) d s\right), i \geq 1$.

\section{Projector for a simple eigenvalue}

In studying Hopf bifurcation it usually requires to consider the projector for a simple eigenvalue. In this section we study the case when $\lambda_{0}$ is a simple eigenvalue of $(A+L)$. That is, $\lambda_{0}$ is a pole of order 1 of the resolvent of $(A+L)$ and the dimension of the eigenspace of $(A+L)$ associated to the eigenvalue $\lambda_{0}$ is 1 .

We know that $\lambda_{0}$ is a pole of order 1 of the resolvent of $(A+L)$ if and only if there exists $\Delta_{-1} \neq 0$, such that

$$
\Delta_{-1}=\lim _{\lambda \rightarrow \lambda_{0}}\left(\lambda-\lambda_{0}\right) \Delta(\lambda)^{-1} .
$$

From Lemma 4.4, we have

$$
\Delta_{-1} \Delta\left(\lambda_{0}\right)=0 \text { and } \Delta\left(\lambda_{0}\right) \Delta_{-1}=0 .
$$

Hence

$$
\begin{gathered}
\Delta_{-1}\left[B y+\widehat{L}\left(e^{\lambda_{0}} \cdot y\right)\right]=\lambda_{0} \Delta_{-1} y, \forall y \in D(B), \\
{\left[B+\widehat{L}\left(e^{\lambda_{0} \cdot I}\right)\right] \Delta_{-1} y=\lambda_{0} \Delta_{-1} y, \forall y \in Y .}
\end{gathered}
$$


So if we assume that $\lambda_{0}$ is a pole of order 1 of $\Delta\left(\lambda_{0}\right)^{-1}$, then $\lambda_{0}$ is simple if and only if $\operatorname{dim}\left[N\left(\Delta\left(\lambda_{0}\right)\right)\right]=1$. Hence,

$$
\Delta_{-1}=\left\langle W_{\lambda_{0}}, .\right\rangle_{Y^{*}, Y} V_{\lambda_{0}} .
$$

Since

$$
\left[B+\widehat{L}\left(e^{\lambda_{0}} \cdot I\right)\right] \Delta_{-1}=\lambda_{0} \Delta_{-1}
$$

we must have $V_{\lambda_{0}} \in D(B)$ and

$$
\Delta\left(\lambda_{0}\right) V_{\lambda_{0}}=0 \Leftrightarrow B V_{\lambda_{0}}+\widehat{L}\left(e^{\lambda_{0}} \cdot V_{\lambda_{0}}\right)=\lambda_{0} V_{\lambda_{0}}
$$

so $V_{\lambda_{0}}$ is an eigenvector of $\Delta\left(\lambda_{0}\right)_{0}$, the part of $\Delta\left(\lambda_{0}\right)$ in $\overline{D(B)}$ (which is the infinitesimal generator of a $C_{0}$-semigroup).

Since $B$ is not densely defined, the characterization of $W_{\lambda_{0}} \in Y^{*}$ is more delicate. First, since

it follows that

$$
\Delta_{-1}\left[B+\widehat{L}\left(e^{\lambda_{0} \cdot} I\right)\right]=\lambda_{0} \Delta_{-1},
$$

$$
\left\langle W_{\lambda_{0}}, B y+\widehat{L}\left(e^{\lambda \cdot y} y\right)\right\rangle_{Y^{*}, Y}=\lambda_{0}\left\langle W_{\lambda_{0}}, y\right\rangle_{Y^{*}, Y}, \forall y \in D(B) .
$$

So $W_{\lambda_{0}} \mid \overline{D(B)}$ (the restriction of $W_{\lambda_{0}}$ to $\left.\overline{D(B)}\right)$ is an adjoint eigenvector of $\Delta\left(\lambda_{0}\right)_{0}$, the part of $\Delta\left(\lambda_{0}\right)$ in $\overline{D(B)}$. But $D(B)$ is not dense (in general) in $Y$, so $\Delta\left(\lambda_{0}\right)^{*}$ is not defined as a linear operator on $Y^{*}$. In order to characterize $W_{\lambda_{0}}$, we observe that $W_{\lambda_{0}}^{0}:=\left.W_{\lambda_{0}}\right|_{\overline{D(B)}} \in N\left(\Delta\left(\lambda_{0}\right)_{0}^{*}\right)$, and by Theorem 3.4

$$
\left\langle W_{\lambda_{0}}, y\right\rangle_{Y^{*}, Y}=\lim _{\lambda \rightarrow+\infty}\left\langle W_{\lambda_{0}}^{0}, \lambda\left(\lambda I-\left(B+\widehat{L}\left(e^{\lambda_{0} \cdot I}\right)\right)\right)^{-1} y\right\rangle_{Y_{0}^{*}, Y_{0}}, \forall y \in Y .
$$

Since $k_{0}=1$ and

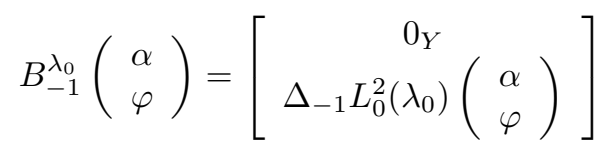

with

$$
L_{0}^{2}\left(\lambda_{0}\right)\left(\begin{array}{c}
\alpha \\
\varphi
\end{array}\right)=e^{\lambda_{0} \theta}\left[\alpha+\varphi(0)+\widehat{L}\left(\int^{0} e^{\lambda_{0}(.-s)} \varphi(s) d s\right)\right],
$$

we can see that $B_{-1}^{\lambda_{0}} B_{-1}^{\lambda_{0}}=B_{-1}^{\lambda_{0}}$ if and only if

$$
\Delta_{-1}=\Delta_{-1}\left[I+\widehat{L}\left(\int^{0} e^{\lambda_{0}} \cdot d s\right)\right] \Delta_{-1}
$$

Therefore, we obtain the following corollary. if

Corollary $5.1 \lambda_{0} \in \sigma((A+L))$ is a simple eigenvalue of $(A+L)$ if and only

$$
\lim _{\lambda \rightarrow \lambda_{0}}\left(\lambda-\lambda_{0}\right)^{2} \Delta(\lambda)^{-1}=0
$$

and

$$
\operatorname{dim}\left[N\left(\Delta\left(\lambda_{0}\right)\right)\right]=1 .
$$

Moreover, the projector on the eigenspace associated to $\lambda_{0}$ is

$$
B_{-1}^{\lambda_{0}}\left(\begin{array}{c}
\alpha \\
\varphi
\end{array}\right)=\left[\begin{array}{c}
0_{Y} \\
e^{\lambda_{0} \theta} \Delta_{-1}\left[\alpha+\varphi(0)+\widehat{L}\left(\int^{0} e^{\lambda_{0}(.-s)} \varphi(s) d s\right)\right]
\end{array},\right.
$$


where

in which

$$
\Delta_{-1}=\left\langle W_{\lambda_{0}}^{*}, .\right\rangle V_{\lambda_{0}}
$$

$$
\begin{gathered}
V_{\lambda_{0}} \in D(B) \backslash\{0\}, \quad B V_{\lambda_{0}}+\widehat{L}\left(e^{\lambda_{0} \cdot} V_{\lambda_{0}}\right)=\lambda_{0} V_{\lambda_{0}}, \quad W_{\lambda_{0}} \in Y^{*} \backslash\{0\}, \\
\left\langle W_{\lambda_{0}}, B y+\widehat{L}\left(e^{\lambda \cdot y} y\right\rangle_{Y^{*}, Y}=\lambda_{0}\left\langle W_{\lambda_{0}}, y\right\rangle_{Y^{*}, Y}, \quad \forall y \in D(B),\right.
\end{gathered}
$$

and

$$
\Delta_{-1}=\Delta_{-1}\left[I+\widehat{L}\left(\int^{0} e^{\lambda_{0} \cdot d s}\right)\right] \Delta_{-1}
$$

\section{Comments on semilinear problems and examples}

In this section we give a few comments and remarks concerning the results obtained in this paper. In order to study the semilinear PFDE

$$
\left\{\begin{array}{l}
\frac{d y(t)}{d t}=B y(t)+\widehat{L}\left(y_{t}\right)+f\left(y_{t}\right), \forall t \geq 0, \\
y_{0}^{\varphi}=\varphi \in C_{B}=\{\varphi \in C([-r, 0] ; Y): \varphi(0) \in \overline{D(B)}\},
\end{array}\right.
$$

we considered the associated abstract Cauchy problem

$$
\frac{d v(t)}{d t}=A v(t)+L(v(t))+F(v(t)), t \geq 0, \quad v(0)=\left(\begin{array}{c}
0_{\mathbb{R}^{n}} \\
\varphi
\end{array}\right) \in \overline{D(A)},
$$

where

$$
F\left(\begin{array}{l}
0 \\
\varphi
\end{array}\right)=\left(\begin{array}{c}
f(\varphi) \\
0
\end{array}\right)
$$

By using Lemma 3.7 we can check that the integrated solutions of (6.2) are the usual solutions of the PFDE (6.1).

Now we are in the position to investigate the properties of the semiflows generated by the PFDE by using the known results on non-densely defined semi-linear Cauchy problems. In particular when $f$ is Lipschitz continuous, from the results of Thieme [45], for each $\varphi \in C_{B}$ we obtain a unique solution $t \rightarrow y^{\varphi}(t)$ on $[-r,+\infty)$ of (6.1), and we can define a nonlinear $C^{0}$-semigroup $\{U(t)\}_{t \geq 0}$ on $C_{B}$ by

$$
U(t) \varphi=y_{t}^{\varphi} .
$$

From the results in Magal [32], one may also consider the case where $f$ is Lipschitz on bounded sets of $C_{B}$. The non-autonomous case has also been considered in Thieme [45] and Magal [32]. We refer to Ezzinbi and Adimy [17] for more results about the existence of solutions using integrated semigroups.

In order to describe the local asymptotic behavior around some equilibrium, we assume that $\bar{y} \in D(B)$ is an equilibrium of the PFDE (6.1), that is,

$$
0=B \bar{y}+L\left(\bar{y} 1_{[-r, 0]}\right)+f\left(\bar{y} 1_{[-r, 0]}\right) .
$$

Then by the stability result of Thieme [45], we obtain the following stability results for PFDE.

Theorem 6.1 (Exponential Stability) Assume that $f: C_{B} \rightarrow \mathbb{R}^{n}$ is continuously differentiable in some neighborhood of $\bar{y} 1_{[-r, 0]}$ and $D f\left(\bar{y} 1_{[-r, 0]}\right)=0$. Assume in addition that

$$
\omega_{0, \text { ess }}\left((A+L)_{0}\right)<0
$$

and each $\lambda \in \mathbb{C}$ such that

$$
N(\Delta(\lambda)) \neq 0
$$


has strictly negative real part. Then there exist $\eta, M, \gamma \in[0,+\infty)$ such that for each $\varphi \in C$ with $\left\|\varphi-\bar{y} 1_{[-r, 0]}\right\|_{\infty} \leq \eta$, the PFDE (6.1) has a unique solution $t \rightarrow y^{\varphi}(t)$ on $[-r,+\infty)$ satisfying

$$
\left\|y_{t}^{\varphi}-\bar{y} 1_{[-r, 0]}\right\|_{\infty} \leq M e^{-\gamma t}\left\|\varphi-\bar{y} 1_{[-r, 0]}\right\|_{\infty}, \forall t \geq 0 .
$$

The above theorem is well known in the context of FDE and PFDE (see, for example, Hale and Verduyn Lunel [22, Corollary 6.1, p. 215] and $\mathrm{Wu}$ [54, Corollary 1.11, p. 71]).

The existence and smoothness of center manifolds was also investigated for abstract non-densely defined Cauchy problems by Magal and Ruan [35]. More precisely, if we denote $\Pi_{c}: X \rightarrow X$ the bounded linear operator of projection

$$
\Pi_{c}=B_{-1}^{\lambda_{1}}+\ldots+B_{-1}^{\lambda_{m}}
$$

where $\left\{\lambda_{1}, \lambda_{2}, \ldots, \lambda_{m}\right\}=\sigma_{C}(A+L):=\{\lambda \in \sigma(A+L): \operatorname{Re}(\lambda)=0\}$. Then

$$
X_{c}=\Pi_{c}(X)
$$

is the direct sum of the generalized eigenspaces associated to the eigenvalues $\left\{\lambda_{1}, \lambda_{2}, \ldots, \lambda_{m}\right\}$.

Moreover,

$$
\Pi_{c}(X) \subset X_{0}
$$

and $\Pi_{c}$ commutes with the resolvent of $(A+L)$. Set

$$
X_{h}=R\left(I-\Pi_{c}\right)\left(\nsubseteq X_{0}\right) .
$$

Then we have the following state space decomposition

$$
X=X_{c} \oplus X_{h} \text { and } X_{0}=X_{0 c} \oplus X_{0 h},
$$

where

$$
X_{0 c}=X_{c} \cap X_{0}=X_{c} \text { and } X_{0 h}=X_{h} \cap X_{0} \neq X_{h} .
$$

Then we can split the original abstract Cauchy problem (6.2) into the following system

$$
\left\{\begin{array}{l}
\frac{d u_{c}(t)}{d t}=(A+L)_{c} u_{c}(t)+\Pi_{c} F\left(u_{c}(t)+u_{h}(t)\right), \\
\frac{d u_{h}(t)}{d t}=(A+L)_{h} u_{h}(t)+\Pi_{h} F\left(u_{c}(t)+u_{h}(t)\right),
\end{array}\right.
$$

where $(A+L)_{c}$, the part of $A+L$ in $X_{c}$, is a bounded linear operator (since $\left.\operatorname{dim}\left(X_{c}\right)<+\infty\right)$, and $(A+L)_{h}$, the part of $A+L$ in $X_{h}$, is a non-densely defined Hille-Yosida operator. So the first equation of (6.3) is an ordinary differential equation and the second equation of (6.3) is a new non-densely defined Cauchy problem with

$$
\sigma\left((A+L)_{h}\right)=\sigma((A+L)) \backslash \sigma_{C}(A+L) .
$$

Assume for simplicity that $f$ is $C^{k}$ in some neighbordhood of the equilibrium $0_{C_{B}}$ and that

$$
f(0)=0 \text { and } D f(0)=0 .
$$

Then we can find (see [35, Theorem 4.21]) a manifold

$$
M=\left\{x_{c}+\psi\left(x_{c}\right): x_{c} \in X_{c}\right\},
$$

where the map $\psi: X_{c} \rightarrow X_{h} \cap \overline{D(A)}$ is $C^{k}$ with

$$
\psi(0)=0, D \psi(0)=0,
$$

and $M$ is locally invariant by the semiflow generated by (6.2). 
More precisely, we can find a neighborhood $\Omega$ of 0 in $C_{B}$ such that if $I \subset \mathbb{R}$ is an interval and $u_{c}: I \rightarrow X_{c}$ is a solution of the ordinary differential equation

$$
\frac{d u_{c}(t)}{d t}=(A+L)_{c} u_{c}(t)+\Pi_{c} F\left(u_{c}(t)+\psi\left(u_{c}(t)\right)\right)
$$

satisfying

$$
u(t):=u_{c}(t)+\psi\left(u_{c}(t)\right) \in \Omega, \forall t \in I,
$$

then $u(t)$ is an integrated solution of $(6.2)$, that is,

$$
u(t)=u(s)+A \int_{s}^{t} u(l) d l+\int_{s}^{t} F(u(l)) d l, \forall t, s \in I \text { with } t \geq s .
$$

Conversely, if $u: \mathbb{R} \rightarrow X_{0}$ is an integrated solution of (6.2) satisfying

$$
u(t) \in \Omega, \forall t \in \mathbb{R},
$$

then $u_{c}(t)=\Pi_{c} u(t)$ is a solution of the ordinary differential equation (6.4). This result leads to the Hopf bifurcation results for PFDE and we refer to $\mathrm{Wu}$ [54] for more results on this subject.

6.1 A reaction-diffusion model with delay ( $B$ is densely defined). Reconsider an example from $\mathrm{Wu}[54]$

$$
\left\{\begin{array}{l}
\frac{\partial u(t, x)}{\partial t}=\varepsilon^{2} \frac{\partial^{2} u(t, x)}{\partial x^{2}}+\delta u(t-r, x), x \in(0,1) \\
\frac{\partial u(t, x)}{\partial x}=0, x=0,1 \\
u(0, .)=u_{0} \in L^{1}(0,1)
\end{array}\right.
$$

where $\alpha \in \mathbb{R}$ and

$$
B \varphi=\varphi^{\prime \prime}
$$

with

$$
D(B)=\left\{\varphi \in W^{2,1}(0,1): \varphi^{\prime}(0)=\varphi^{\prime}(1)=0\right\} .
$$

We compute the projectors. Firstly, we have the following lemma.

Lemma 6.2 The linear operator $B: D(B) \subset L^{1}(0,1) \rightarrow L^{1}(0,1)$ is the infinitesimal generator of an analytic semigroup $\left\{T_{B}(t)\right\}_{t \geq 0}$ on $L^{1}(0,1)$. Moreover, we have the following properties

(a) $T_{B}(t)$ is compact for each $t>0$.

(b) $T_{B}(t) L_{+}^{1}(0,1) \subset L_{+}^{1}(0,1)$ for each $t \geq 0$.

(c) $\sigma(B)=\sigma_{P}(B)=\left\{\lambda_{n}=-(n \pi \varepsilon)^{2}: n \geq 0\right\}$.

(d) For each $n \geq 0, \lambda_{n}=-(n \pi \varepsilon)^{2}$ is a simple eigenvalue of $B$, the projector on the eigenspace associated to $\lambda_{n}$ is given by

$$
\Pi_{n}(\varphi)(x)= \begin{cases}\int_{0}^{1} \varphi(y) d y, & \text { if } n=0 \\ 2\left(\int_{0}^{1} \cos (n \pi y) \varphi(y) d y\right) \cos (n \pi x), & \text { if } n \geq 1 .\end{cases}
$$

Here

$$
C_{B}=C=C\left([-r, 0], L^{1}(0,1)\right)
$$

and the linear operator $\widehat{L}: C \rightarrow L^{1}(0,1)$ is defined by

$$
\widehat{L}(\varphi)=\delta \varphi(-r) \text {. }
$$


Moreover, by applying Lemma 3.10-(b), we obtain

$$
\omega_{0, \mathrm{ess}}\left((A+L)_{0}\right)=-\infty
$$

The characteristic function is given by

$$
\Delta(\lambda) x=\lambda x-B x-\delta e^{-\lambda r} x,
$$

so $\Delta(\lambda)$ is invertible if and only if $\lambda-\delta e^{-\lambda r} \notin \sigma(B)$. Thus,

$$
\sigma\left((A+L)_{0}\right)=\left\{\lambda \in \mathbb{C}: \lambda-\delta e^{-\lambda r} \in \sigma(B)\right\} .
$$

Let $\widehat{\lambda} \in \sigma\left((A+L)_{0}\right)$ be given and let $n_{0} \geq 0$ such that

$$
\widehat{\lambda}-\delta e^{-\widehat{\lambda} r}=\lambda_{n_{0}},
$$

where $\lambda_{n_{0}} \in \sigma(B)$.

Now we prove the following result.

Proposition 6.3 Suppose that

$$
\gamma=\left.\frac{d}{d \lambda}\left(\lambda-\delta e^{-\lambda r}-\lambda_{n_{0}}\right)\right|_{\lambda=\widehat{\lambda}} \neq 0 .
$$

Then the eigenvalue $\hat{\lambda}$ is a simple eigenvalue of $(A+L)$. Moreover, we have

$$
\Delta_{-1}=\gamma^{-1} \Pi_{n_{0}}
$$

and

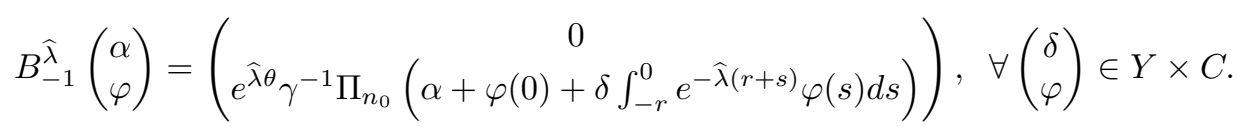

Proof Let us first notice that

$$
N(\Delta(\widehat{\lambda}))=N\left(\lambda_{n_{0}} I-B\right) .
$$

Thus, due to Lemma 6.2 , it is a one-dimensional space. Now we compute $\Delta_{-1}$. For $\lambda \neq \hat{\lambda}$ with $|\lambda-\widehat{\lambda}|$ small enougth,

$$
\begin{aligned}
\Delta(\lambda)^{-1} & =\left(\lambda I-\delta e^{-\lambda r} I-B\right)^{-1} \\
& =\left(\lambda I-\delta e^{-\lambda r} I-B\right)^{-1} \Pi_{n_{0}}+\left(\lambda I-\delta e^{-\lambda r} I-B\right)^{-1}\left(I-\Pi_{n_{0}}\right) \\
& =\frac{1}{\lambda-\delta e^{-\lambda r}-\lambda_{n_{0}}} \Pi_{n_{0}}+\left(\lambda I-\delta e^{-\lambda r} I-B\right)^{-1}\left(I-\Pi_{n_{0}}\right)
\end{aligned}
$$

and $\left(\lambda I-\delta e^{-\lambda r} I-B\right)^{-1}\left(I-\Pi_{n_{0}}\right)$ is bounded in the norm of operators for $\lambda$ close enough to $\hat{\lambda}$. Since $\gamma \neq 0$, it follows that $\widehat{\lambda}$ is a pole of order 1 of $\Delta(\lambda)^{-1}$ and we have

$$
\lim _{\lambda \rightarrow \widehat{\lambda}}(\lambda-\widehat{\lambda}) \Delta(\lambda)^{-1}=\gamma^{-1} \Pi_{n_{0}}
$$

Moreover, we can easily get that

$$
\lim _{\lambda \rightarrow \widehat{\lambda}}(\lambda-\widehat{\lambda})^{2} \Delta(\lambda)^{-1}=0 .
$$

Thus, Corollary 5.1 applies and also provides the formula for the generalized projector. This completes the proof of the proposition. 
Remark 6.4 If $\gamma=0$, then $\alpha \neq 0$ and we obtain that $\widehat{\lambda}$ is a pole of order two of $\Delta(\lambda)^{-1}$. Indeed, we can easily obtain that

$$
\lim _{\lambda \rightarrow \widehat{\lambda}}(\lambda-\widehat{\lambda}) \Delta(\lambda)^{-1}=\frac{2}{\widehat{\gamma}} \Pi_{n_{0}},
$$

where $\widehat{\gamma}=-\delta \tau^{2} e^{-\hat{\lambda} \tau} \neq 0$, while

$$
\lim _{\lambda \rightarrow \widehat{\lambda}}(\lambda-\widehat{\lambda})^{2} \Delta(\lambda)^{-1}=0 .
$$

Then we can derive the expression for the corresponding eigenprojector according to Proposition 4.5.

6.2 An age-structured model with delay ( $B$ is non-densely defined). By taking into account the time $r>0$ between the reproduction and the birth of individuals, one may introduce the following (simplified) age-structured model with delay

$$
\left\{\begin{array}{l}
\frac{\partial u}{\partial t}+\frac{\partial u}{\partial a}=-\mu u(t, a), a \geq 0, \\
u(t, 0)=\int_{0}^{+\infty} b(a) u(t-r, a) d a \\
u(0, .)=u_{0} \in L^{1}(0,+\infty),
\end{array}\right.
$$

where $r>0, \mu>0$, and $b \in L_{+}^{\infty}(0,+\infty)$.

In this case we set

$$
Y=\mathbb{R} \times L^{1}(0,+\infty)
$$

endowed with the usual product norm

$$
\left\|\left(\begin{array}{c}
\alpha \\
\varphi
\end{array}\right)\right\|=|\alpha|+\|\varphi\|_{L^{1}(0,+\infty)} .
$$

Define $B: D(B) \subset Y \rightarrow Y$ by

$$
B\left(\begin{array}{c}
0 \\
\varphi
\end{array}\right)=\left(\begin{array}{c}
-\varphi(0) \\
-\varphi^{\prime}-\mu \varphi
\end{array}\right)
$$

where

$$
D(B)=\{0\} \times W^{1,1}(0,+\infty)
$$

Then it is clear that

$$
Y_{0}=\overline{D(B)}=\{0\} \times L^{1}(0,+\infty),
$$

so $B$ is non-densely defined. Also define

$$
\widetilde{L}\left(\left(\begin{array}{c}
0 \\
\varphi
\end{array}\right)\right)=\left(\begin{array}{c}
\int_{0}^{+\infty} b(a) \varphi(a) d a \\
0
\end{array}\right) .
$$

Then by identifying $u(t)$ and $v(t)=\left(\begin{array}{c}0 \\ u(t)\end{array}\right)$, the equation (6.6) can be rewritten as the following abstract Cauchy problem

$$
\left\{\begin{array}{l}
\frac{d v(t)}{d t}=B v(t)+\widetilde{L}(v(t-r)), t \geq 0 \\
v_{0}=\varphi \in C_{B}
\end{array}\right.
$$

Here

$$
C_{B}=\left\{\left(\begin{array}{c}
\alpha(.) \\
\varphi(.)
\end{array}\right) \in C([-r, 0], Y): \alpha(0)=0\right\}
$$


and the operator $\widehat{L}: C_{B} \rightarrow Y$ is defined by

$$
\widehat{L}\left(\begin{array}{c}
\alpha(.) \\
\varphi(.)
\end{array}\right)=\widetilde{L}\left(\left(\begin{array}{c}
0 \\
\varphi(-r)
\end{array}\right)\right)=\left(\begin{array}{c}
\alpha \int_{0}^{+\infty} b(a) \varphi(-r)(a) d a \\
0
\end{array}\right)
$$

Now we can explicitly compute the resolvent of the operator $B$. Indeed, we have for each $\lambda \in \mathbb{C}$ with $\operatorname{Re}(\lambda)>-\mu$ that

$$
\begin{aligned}
& (\lambda I-B)^{-1}\left(\begin{array}{c}
\alpha \\
\psi
\end{array}\right)=\left(\begin{array}{c}
0 \\
\varphi
\end{array}\right) \\
& \Leftrightarrow \varphi(a)=e^{-(\lambda+\mu) a} \alpha+\int_{0}^{a} e^{-(\lambda+\mu)(a-l)} \psi(l) d l .
\end{aligned}
$$

Next, since the operator $\widehat{L}$ is a one-dimensional rank operator we obtain by using Lemma 3.10-(a) that

$$
\left.\omega_{0, \mathrm{ess}}\left((A+L)_{0}\right)\right) \leq \omega_{0, \mathrm{ess}}\left(A_{0}\right) \leq \omega_{0, \mathrm{ess}}\left(B_{0}\right) \leq-\mu .
$$

Setting

$$
\Omega=\{\lambda \in \mathbb{C}: \operatorname{Re}(\lambda)>-\mu\}
$$

and using Lemma 4.4, we obtain that

$$
\sigma(A+L) \cap \Omega=\{\lambda \in \Omega: \quad \lambda \text { is a pole of } \Delta(\lambda)\},
$$

where the operator $\Delta(\lambda): D(B) \subset Y \rightarrow Y$ is defined by

$$
\Delta(\lambda)=\lambda I-B-e^{-\lambda r} \widetilde{L} .
$$

We now derive the characteristic equation for this problem.

Define a characteristic function $\widehat{\Delta}: \Omega \rightarrow \mathbb{C}$ by

$$
\widehat{\Delta}(\lambda):=1-e^{-\lambda r} \int_{0}^{+\infty} b(a) e^{-(\mu+\lambda) a} d a, \forall \lambda \in \Omega .
$$

By using Lemmas 4.1 and 4.2, we obtain the following result.

Lemma 6.5 We have

$$
\sigma(A+L) \cap \Omega=\{\lambda \in \Omega: \Delta(\lambda) \text { is not invertible }\}=\{\lambda \in \Omega: \widehat{\Delta}(\lambda)=0\} .
$$

Moreover, for each $\lambda \in \sigma(A+L) \cap \Omega$,

$$
\begin{aligned}
& \Delta(\lambda)^{-1}\left(\begin{array}{l}
\alpha \\
\psi
\end{array}\right)=\left(\begin{array}{l}
0 \\
\varphi
\end{array}\right) \\
& \Leftrightarrow \varphi(a)=\left[e^{-(\lambda+\mu) a} \alpha+\int_{0}^{a} e^{-(\lambda+\mu)(a-l)} \psi(l) d l\right] \\
& +\widehat{\Delta}(\lambda)^{-1} e^{-(\lambda+\mu) a} e^{-\lambda r} \int_{0}^{+\infty} b(\sigma)\left[e^{-(\lambda+\mu) \sigma} \alpha+\int_{0}^{\sigma} e^{-(\lambda+\mu)(\sigma-l)} \psi(l) d l\right] d \sigma \\
& (\lambda I-(A+L))^{-1}\left(\begin{array}{c}
\alpha \\
\varphi
\end{array}\right)=\left(\begin{array}{c}
0_{Y} \\
\psi
\end{array}\right) \\
& \Leftrightarrow \\
& \psi(\theta)=\int_{\theta}^{0} e^{\lambda(\theta-s)} \varphi(s) d s+e^{\lambda \theta} \Delta(\lambda)^{-1}\left[\alpha+\varphi(0)+\widehat{L}\left(\int_{0}^{0} e^{\lambda(.-s)} \varphi(s) d s\right)\right] .
\end{aligned}
$$

In the case of simple eigenvalues we obtain the following result.

Proposition 6.6 Assume that $\sigma(A+L) \cap \Omega \neq \emptyset$. Let $\lambda_{0} \in \sigma(A+L) \cap \Omega$. If

$$
\frac{d \widehat{\Delta}\left(\lambda_{0}\right)}{d \lambda}=r+e^{-\lambda_{0} r} \int_{0}^{+\infty} a b(a) e^{-\left(\mu+\lambda_{0}\right) a} d a \neq 0,
$$


then $\lambda_{0}$ is a pole of order 1 of $(\lambda I-(A+L))^{-1}$ and

$$
B_{-1}^{\widehat{\lambda}}\left(\begin{array}{l}
\alpha \\
\varphi
\end{array}\right)=\left(e^{\lambda \theta} \Delta_{-1}\left[\alpha+\varphi(0)+\widehat{L}\left(\int_{.}^{0} e^{\lambda(.-s)} \varphi(s) d s\right)\right]\right)
$$

where the linear operator $\Delta_{-1}=\lim _{\lambda \rightarrow \lambda_{0}}\left(\lambda-\lambda_{0}\right) \Delta(\lambda)^{-1}$ is defined by

$$
\begin{aligned}
& \Delta_{-1}\left(\begin{array}{l}
\alpha \\
\psi
\end{array}\right)=\left(\begin{array}{l}
0 \\
\varphi
\end{array}\right) \\
& \Leftrightarrow \varphi(a)={\frac{d \widehat{\Delta}\left(\lambda_{0}\right)}{d \lambda}}^{-1} e^{-\left(\lambda_{0}+\mu\right) a} e^{-\lambda_{0} r} \int_{0}^{+\infty} b(\sigma)\left[e^{-\left(\lambda_{0}+\mu\right) \sigma} \alpha+\int_{0}^{\sigma} e^{-\left(\lambda_{0}+\mu\right)(\sigma-l)} \psi(l) d l\right] d \sigma .
\end{aligned}
$$

\section{References}

[1] M. Adimy, Bifurcation de Hopf locale par des semi-groupes intégrés, C. R. Acad. Sci. Paris Série I 311 (1990), 423-428.

[2] M. Adimy, Integrated semigroups and delay differential equations, J. Math. Anal. Appl. 177 (1993), 125-134.

[3] M. Adimy and O. Arino, Bifurcation de Hopf globale pour des équations à retard par des semi-groupes intégrés, C. R. Acad. Sci. Paris Série I 317 (1993), 767-772.

[4] M. Adimy, K. Ezzinbi and J. Wu, Center manifold and stability in critical cases for some partial functional differential equations, Int. J. Evol. Equ. 2 (2007), 47-73.

[5] W. Arendt, Vector valued Laplace transforms and Cauchy problems, Israel J. Math. 59 (1987), 327-352.

[6] W. Arendt, C. J. K. Batty, M. Hieber, and F. Neubrander, Vector-Valued Laplace Transforms and Cauchy Problems, Birkhäuser, Basel, 2001.

[7] O. Arino, M. L. Hbid and E. Ait Dads, Delay Differential Equations and Applications, Springer, Berlin, 2006.

[8] O. Arino and E. Sanchez, A variation of constants formula for an abstract functionaldifferential equation of retarded type, Differential Integral Equations 9 (1996), 1305-1320.

[9] O. Arino and E. Sánchez, A theory of linear delay differential equations in infinite dimensional spaces, in "Delay Differential Equations with Application", O. Arino, M. Hbid and E. Ait Dads (eds.), NATO Science Series II: Mathematics, Physics and Chemistry, Vol. 205, Springer, Berlin, 2006, pp. 287-348.

[10] H. Brezis, Analyse Fonctionnelle, Dunod, Paris, 2005

[11] F. E. Browder, On the spectral theory of elliptic differential operators, Math. Ann. 142 (1961), 22-130.

[12] S. Busenberg and W. Huang, Stability and Hopf bifurcation for a population delay model with diffusion effects, J. Differential Equations 124 (1996), 80-107.

[13] O. Diekmann, P. Getto and M. Gyllenberg, Stability and bifurcation analysis of Volterra functional equations in the light of suns and stars, SIAM J. Math. Anal. 34 (2007), 10231069.

[14] O. Diekmann, S. A. van Gils, S. M. Verduyn Lunel, and H.-O. Walther, Delay Equations, Function-, Complex-, and Nonlinear Analyiss, Springer-Verlag, New York, 1995.

[15] A. Ducrot, Z. Liu and P. Magal, Essential growth rate for bounded linear perturbation of non-densely defined Cauchy problems, J. Math. Anal. Appl. 341 (2008), 501-518.

[16] K.-J. Engel and R. Nagel, One Parameter Semigroups for Linear Evolution Equations, Springer-Verlag, New York, 2000.

[17] K. Ezzinbi and M. Adimy, The basic theory of abstract semilinear functional differential equations with non-dense domain, in "Delay Differential Equations with Application", O. Arino, M. Hbid and E. Ait Dads (eds.), NATO Science Series II: Mathematics, Physics and Chemistry, Vol. 205, Springer, Berlin, 2006, pp. 349-400.

[18] T. Faria, Normal forms and Hopf bifurcation for partial differential equations with delays, Trans. Amer. Math. Soc. 352 (2000), 2217-2238.

[19] T. Faria, W. Huang and J. Wu, Smoothness of center manifolds for maps and formal adjoints for semilinear FDEs in general Banach spaces, SIAM J. Math. Anal. 34 (2002), 173-203.

[20] W. E. Fitzgibbon, Semilinear functional differential equations in Banach space, J. Differential Equations 29 (1978), 1-14. 
[21] M. V. S. Frasson and S. M. Verduyn Lunel, Large time behaviour of linear functional differential equations, Integr. Equ. Oper. Theory 47 (2003), 91-121.

[22] J. K. Hale and S. M. Verduyn Lunel, Introduction to Functional Differential Equations, Springer-Verlag, New York, 1993.

[23] B. D. Hassard, N. D. Kazarinoff and Y.-H. Wan, Theory and Applications of Hopf Bifurcaton, Londonn Math. Soc. Lect. Note Ser. 41, Cambridge Univ. Press, Cambridge, 1981.

[24] M. A. Kaashoek and S. M. Verduyn Lunel, Characteristic matrices and spectral properties of evolutionary systems, Trans. Amer. Math. Soc. 334 (1992), 479-517.

[25] F. Kappel, Linear autonomous functional differential equations, in "Delay Differential Equations with Application", O. Arino, M. Hbid and E. Ait Dads (eds.), NATO Science Series II: Mathematics, Physics and Chemistry, Vol. 205, Springer, Berlin, 2006, pp. 41-134.

[26] H. Kellermann and M. Hieber, Integrated semigroups, J. Funct. Anal. 84 (1989), 160-180.

[27] X. Lin, J. W.-H. So and J. Wu, Centre manifolds for partial differential equations with delays, Proc. Roy. Soc. Edinburgh 122A (1992), 237-254.

[28] Z. Liu, P. Magal and S. Ruan, Projectors on the generalized eigenspaces for functional differential equations using integrated semigroups, J. Differential Equations 244 (2008), 1784-1809.

[29] Z. Liu, P. Magal, S. Ruan and J. Wu, Normal forms for semilinear equations with non-dense domain, Part I: Computation of the reduced system, submitted.

[30] R. H. Martin and H. L. Smith, Abstract functional-differential equations and reactiondiffusion systems, Trans. Amer. Math. Soc. 321 (1990), 1-44.

[31] R. H. Martin and H. L. Smith, Reaction-diffusion systems with time delays: monotonicity, invariance, comparison and convergence, J. Reine Angew. Math. 413 (1991), 1-35.

[32] P. Magal, Compact attractors for time periodic age-structured population models, Electr. J. Differential Equations 2001 (2001), No. 65, 1-35.

[33] P. Magal and S. Ruan, On integrated semigroups and age structured models in $L^{p}$ spaces, Differential Integral Equations 20 (2007), 197-239.

[34] P. Magal and S. Ruan, On semilinear Cauchy problems with non-dense domain, Adv. Differential Equations 14 (2009), 1041-1084.

[35] P. Magal and S. Ruan, Center manifold theorem for semilinear equations with non-dense domain and applications to Hopf bifurcations in age structured models, Mem. Amer. Math. Soc. Vol. 202, 2009, No. 951.

[36] M. C. Memory, Bifurcation and asymptotic behavior of solutions of a delay-differential equation with diffusion, SIAM J. Math. Anal. 20 (1989), 533-546.

[37] M. C. Memory, Stable ans unstable manifolds for partial functional differential equations, Nonlinear Anal. 16 (1991), 131-142.

[38] M. E. Parrott, Linearized stability and irreducibility for a functional-differential equation, SIAM J. Math. Anal. 23 (1992), 649-661.

[39] A. Pazy, Semigroups of Linear Operators and Applications to Partial Differential Equations, Springer-Verlag, New-York, 1983.

[40] A. Rhandi, Extrapolated methods to solve non-autonomous retarded partial dierential equations, Studia Math. 126 (1997), 219-233.

[41] S. Ruan, J. Wei and J. Wu, Bifurcation from a homoclinic orbit in partial functional differential equations, Discret. Contin. Dynam. Syst. 9A (2003), 1293-1322.

[42] S. Ruan and W. Zhang, Exponential dichotomies, the Fredholm alternative, and transverse homoclinic orbits in partial functional differential equations, J. Dynamics Differential Equations 17 (2005), 759-777.

[43] W. M. Ruess, Existence and stability of solutions to partial functional differential equations with delay, Adv. Differential Equations 4 (1999), 843-867.

[44] W. M. Ruess, Linearized stability for nonlinear evolution equations, J. Evol. Equ. 3 (2003), 361-373.

[45] H. R. Thieme, Semiflows generated by Lipschitz perturbations of non-densely defined operators, Differential Integral Equations 3 (1990), 1035-1066.

[46] H. R. Thieme, Integrated semigroups and integrated solutions to abstract Cauchy problems, J. Math. Anal. Appl. 152 (1990), 416-447.

[47] H. R. Thieme, Quasi-compact semigroups via bounded perturbation, in "Advances in Mathematical Population Dynamics-Molecules, Cells and Man", Ser. Math. Biol. Med. Vol. 6, World Sci. Publishing, River Edge, NJ, 1997, pp. 691-711. 
[48] C. C. Travis and G. F. Webb, Existence and stability for partial functional differential equations, Trans. Amer. Math. Soc. 200 (1974), 395-418.

[49] C. C. Travis and G. F. Webb, Existence, stability, and compactness in the $\alpha$-norm for partial functional differential equations, Trans. Amer. Math. Soc. 240 (1978), 129-143.

[50] S. M. Verduyn Lunel, Spectral theory for delay equations, in "Systems, Approximation, Singular Integral Operators, and Related Topics", A. A. Borichev and N. K. Nikolski (eds.), Operator Theory: Advances and Applications, Vol. 129, Birkhäuser, 2001, pp. 465-508.

[51] G. F. Webb, Functional differential equations and nonlinear semigroups in $L^{p}$-spaces, J. Differential Equations 20 (1976), 71-89.

[52] G. F. Webb, Theory of Nonlinear Age-Dependent Population Dynamics, Marcel Dekker, New York, 1985

[53] G. F. Webb, An operator-theoretic formulation of asynchronous exponential growth, Trans. Amer. Math. Soc. 303 (1987), 155-164.

[54] J. Wu, Theory and Applications of Partial Differential Equations, Springer-Verlag, New York, 1996.

[55] K. Yoshida, The Hopf bifurcation and its stability for semilinear diffusion equations with time delay arising in ecology, Horoshima Math. J. 12 (1982), 321-348. 